\title{
WILSON BASES FOR GENERAL TIME-FREQUENCY LATTICES
}

\author{
GITTA KUTYNIOK* AND THOMAS STROHMER ${ }^{\dagger}$
}

\begin{abstract}
Motivated by a recent generalization of the Balian-Low theorem and by new research in wireless communications we analyze the construction of Wilson bases for general time-frequency lattices. We show that orthonormal Wilson bases for $L^{2}(\mathbb{R})$ can be constructed for any time-frequency lattice whose volume is $\frac{1}{2}$. We then focus on the spaces $\ell^{2}(\mathbb{Z})$ and $\mathbb{C}^{L}$ which are the preferred settings for numerical and practical purposes. We demonstrate that with a properly adapted definition of Wilson bases the construction of orthonormal Wilson bases for general time-frequency lattices also holds true in these discrete settings. In our analysis we make use of certain metaplectic transforms. Finally we discuss some practical consequences of our theoretical findings.
\end{abstract}

Key words. Wilson basis, metaplectic transform, Gabor frame, Schrödinger representation, time-frequency lattice

AMS subject classifications. 94A12, 42C15

1. Introduction. Gabor systems have become a popular tool, both in theory and in applications, e.g., see [14, 20, 15. However one drawback is that due to the Balian-Low theorem it is impossible to construct (orthogonal) Gabor bases for $L^{2}(\mathbb{R})$ with good time-frequency localization [20]. In [35, 11] it has been shown that a modification of Gabor bases, so-called Wilson bases provide a means to circumvent the Balian-Low theorem. Indeed, there exist orthogonal Wilson bases for $L^{2}(\mathbb{R})$ whose basis functions have exponential decay in time and frequency. These Wilson bases can be constructed from certain tight Gabor frames with redundancy 2.

Gabor frames are usually associated with rectangular time-frequency lattices, but they can also be defined for general non-separable lattices, see e.g. [12, 19, 13, 30]. Recently it has been shown that such a generalization of Gabor frames to general timefrequency lattices does not enable us to overcome the Balian-Low theorem 21, 2]. This leads naturally to the question if it is possible to extend the construction of Wilson bases to general time-frequency lattices.

Another motivation for the research presented in this paper has its origin in wireless communication. Orthogonal frequency division multiplexing (OFDM) is a wireless transmission technology employing a set of transmission functions which is usually associated with a rectangular time-frequency lattice [16]. The connection to Gabor theory is given by the fact that the collection of transmission pulses in OFDM can be interpreted as a Gabor system, see [29, 32]. The density of the associated rectangular time-frequency lattice can be seen as a measure of the spectral efficiency in terms of number of bits transmitted per Hertz per second. The necessary condition of linear independence of the transmission functions implies that we are dealing with either an undersampled or a critically sampled Gabor system.

For wireless channels that are time-dispersive (due to multipath) and frequencydispersive (due to the Doppler effect) good time-frequency localization of the transmission pulses is essential to mitigate the interferences caused by the dispersion of the channel [29] 32]. The ideal set of transmission pulses should therefore possess (i) good time-frequency localization and (ii) maximize the spectral efficiency, i.e.,

\footnotetext{
*Institute of Mathematics, University of Paderborn, 33095 Paderborn, Germany; gittak@upb.de. G.K. acknowledges support from Forschungspreis 2003 der Universität Paderborn.

${ }^{\dagger}$ Department of Mathematics, University of California, Davis, CA 95616-8633, USA; strohmer@math.ucdavis.edu. T.S. acknowledges support from NSF DMS grant 0208568.
} 
the transmission functions should correspond to a (critically sampled) Gabor basis for $L^{2}(\mathbb{R})$. As we know, the Balian-Low theorem prohibits these conditions to be fulfilled simultaneously.

Recently it has been shown that in case of time-frequency dispersive channels the performance of OFDM can be improved when using general time-frequency lattices, in particular hexagonal-type lattices 33 . In a nutshell, lattices that are adapted to the shape of the Wigner distribution of the transmission pulses allow for a better "packing" of the time-frequency plane, which in turn can be used to either achieve higher data rates or to improve interference robustness of the associated so-called Lattice-OFDM system.

One variation of OFDM (for rectangular lattices) is called Offset-QAM (OQAM) OFDM, it corresponds to using a Wilson basis as set of transmission functions [5]. OQAM-OFDM achieves maximal spectral efficiency and allows for transmission functions with good time-frequency localization. As in the case of standard OFDM it would be potentially useful for time-frequency dispersive channels to extend OQAMOFDM to general time-frequency lattices in order to improve the robustness of OQAMOFDM against interference even further. Thus we again arrive at the problem of constructing Wilson bases for general non-separable time-frequency lattices.

Yet another motivation comes from filter bank theory, more precisely cosinemodulated filter banks [7. We know that discrete-time Wilson bases correspond to a special class of cosine-modulated filter banks (see [7]). In light of the improvements gained by using general time-frequency lattices in OFDM [33, it would be interesting to analyze if the construction of cosine-modulated filter banks can be extended to general time-frequency lattices. A positive answer to this question might lead to a more efficient encoding of signals and images.

Since our goal to construct Wilson bases for general time-frequency lattices is in part motivated by applied problems and since any numerical implementation of Wilson bases is based on a discrete model, our analysis will not only concern $L^{2}(\mathbb{R})$ but also comprise the spaces $\ell^{2}(\mathbb{Z})$ and $\mathbb{C}^{L}$. Furthermore, $\ell^{2}(\mathbb{Z})$ is the appropriate setting when Wilson bases are utilized as filter banks, since in this case one deals with sampled, thus discrete-time, signals.

1.1. Notation. We assume that the reader is familiar with the theory of Gabor frames and refer to 20 for background and details.

A lattice $\Lambda$ in $\mathbb{R}^{d}$ is a discrete subgroup with compact quotient, i.e., there exists a matrix $A \in G L(d, \mathbb{R})$ such that $\Lambda=A \mathbb{Z}^{d}$. The matrix $A$ is called the (non-unique) generator matrix for $\Lambda$. The volume of $\Lambda$ is $\operatorname{vol}(\Lambda)=|\operatorname{det}(A)|$. Two lattices, which play a crucial role in OFDM design (see [33), are the rectangular lattice $\Lambda_{R}$ and the hexagonal lattice $\Lambda_{H}$. A generator matrix for $\Lambda_{R}$ is given by

$$
A_{R}=\left[\begin{array}{cc}
T & 0 \\
0 & F
\end{array}\right]
$$

and a generator matrix for $\Lambda_{H}$ is given by

$$
A_{H}=\left[\begin{array}{cc}
\frac{\sqrt{2}}{\sqrt[4]{3}} T & \frac{\sqrt{2}}{2 \sqrt[4]{3}} T \\
0 & \frac{\sqrt[4]{3}}{\sqrt{2}} F
\end{array}\right],
$$

where $T, F>0$. An easy calculation shows that both lattices $\Lambda_{R}$ and $\Lambda_{H}$ have the same volume $T F$. A normal form for matrices, which we will use in the following, 
is the so-called Hermite normal form $[22]$. We say that a matrix $A=\left[\begin{array}{ll}a & b \\ c & d\end{array}\right]$ is in Hermite normal form, if $c=0, a, d>0$, and $0 \leq b<a$. For example both matrices $A_{R}$ and $A_{H}$ are in Hermite normal form.

For $(x, y) \in \mathbb{R}^{2}$ and $g \in L^{2}(\mathbb{R})$, let $g_{x, y}$ be defined by

$$
g_{x, y}(t)=e^{2 \pi i y t} g(t-x) .
$$

We denote by $\mathcal{G}(g, \Lambda)$ the system of functions given by

$$
g_{\lambda, \mu}(t)=g(t-\lambda) e^{2 \pi i t \mu}, \quad(\lambda, \mu) \in \Lambda .
$$

As usual, the redundancy of $\mathcal{G}(g, \Lambda)$ is given by $\frac{1}{\operatorname{vol}(\Lambda)}$.

As in [20], we define the Schrödinger representation $\rho: \mathbb{H} \rightarrow \mathcal{U}\left(L^{2}(\mathbb{R})\right)$ by

$$
\rho(x, y, z) g(t)=e^{2 \pi i z} e^{-\pi i x y} e^{2 \pi i y t} g(t-x) .
$$

Note that

$$
g_{x, y}=e^{\pi i x y} \rho(x, y, 1) g .
$$

Furthermore, we use the following notation from [20] (with slight changes):

$$
\mathcal{J}=\left[\begin{array}{cc}
0 & -1 \\
1 & 0
\end{array}\right], \quad \mathcal{B}_{b}=\left[\begin{array}{cc}
b & 0 \\
0 & \frac{1}{b}
\end{array}\right], \quad \mathcal{C}_{c}=\left[\begin{array}{ll}
1 & 0 \\
c & 1
\end{array}\right] .
$$

$\mathcal{F}$ and ${ }^{\wedge}$ denote the Fourier transform, $\mathcal{F}^{-1}$ and ${ }^{\vee}$ denote the inverse Fourier transform. The dilation is given by $\mathcal{D}_{b} f(t)=|b|^{\frac{1}{2}} f(b t)$ and the "chirp" operator is defined via $\mathcal{N}_{c} f(t)=e^{-\pi i c t^{2}} f(t)$, where $b, c \in \mathbb{R}$ and $f \in L^{2}(\mathbb{R})$.

Metaplectic transforms will turn out to be a very useful tool in our analysis. For the study of the discrete and finite case, we need a result on metaplectic transforms from [26]. Since this thesis is not easy to access, we present the result here in the slightly weaker version we will use, together with the necessary definitions and notations. The result will be stated in the situation of a general locally compact abelian group $G$ with dual group $\widehat{G}$, group multiplication denoted by + , and action of $\widehat{G}$ on $G$ denoted by $\langle x, \chi\rangle$ for $x \in G$ and $\chi \in \widehat{G}$. The cases we are interested in later on are $G=\mathbb{Z}$ and $G=\mathbb{Z}_{L}$. We will usually write a metaplectic transform $\sigma$ in the matrix notation $\sigma=\left[\begin{array}{ll}\alpha & \beta \\ \gamma & \delta\end{array}\right] \in \operatorname{Hom}(G \times \widehat{G})$, which means that $\alpha \in \operatorname{Hom}(G)$, $\beta \in \operatorname{Hom}(\widehat{G}, G), \gamma \in \operatorname{Hom}(G, \widehat{G})$, and $\delta \in \operatorname{Hom}(\widehat{G})$. Then the adjoint $\sigma^{*}$ is defined by $\left\langle(x, \chi), \sigma^{*}(y, \pi)\right\rangle=\langle\sigma(x, \chi),(y, \pi)\rangle$ for all $(x, \chi),(y, \pi) \in G \times \widehat{G}$. Let $\eta$ be defined by $\eta=\left[\begin{array}{cc}0 & -I_{\widehat{G}} \\ I_{G} & 0\end{array}\right] \in \operatorname{Hom}(G \times \widehat{G}, \widehat{G} \times G)$, where the above definition concerning the matrix notation has to be adapted in an obvious way, and where $I_{G}$ and $I_{\widehat{G}}$ denote the identity on $G$ and $\widehat{G}$, respectively. Then $\sigma$ is called symplectic, if $\sigma^{*} \eta \sigma=\eta$. If $\zeta \in \operatorname{Hom}(G \times \widehat{G}, \widehat{G} \times G)$, then $\psi$ is a second degree character of $G \times \widehat{G}$ associated to $\zeta$, if $\psi((x, \chi)+(y, \pi))=\psi(x, \chi) \psi(y, \pi)\langle(x, \chi), \zeta(y, \pi)\rangle$ for all $(x, \chi),(y, \pi) \in G \times \widehat{G}$. Moreover, for $(x, \chi) \in G \times \widehat{G}$ and $g \in L^{2}(G)$, let $g_{x, \chi}$ be defined by

$$
g_{x, \chi}(t)=\chi(t) g(t-x),
$$

which generalizes the previous definition. 
We can now state the version of [26] Theorem 1.1.28], which we will employ in Sections 3 and 4

TheOREM 1.1. Let $\sigma:=\left[\begin{array}{ll}\alpha & \beta \\ \gamma & \delta\end{array}\right] \in \operatorname{Hom}(G \times \widehat{G})$ be symplectic and let $\psi_{\zeta}$ be a second degree character of $G \times \widehat{G}$ associated to

$$
\zeta:=\sigma^{*}\left[\begin{array}{cc}
0 & 0 \\
I_{G} & 0
\end{array}\right] \sigma-\left[\begin{array}{cc}
0 & 0 \\
I_{G} & 0
\end{array}\right] \in \operatorname{Hom}(G \times \widehat{G}, \widehat{G} \times G) .
$$

If $U$ is defined by

$$
U f(t):=\int_{\widehat{G}} f(\alpha t+\beta \omega) \psi_{\zeta}^{-1}(t, \omega) d \omega
$$

then we have

$$
(U f)_{x, \chi}(t)=\psi_{\zeta}^{-1}(x, \chi) U f_{\sigma(x, \chi)}(t), \quad(x, \chi) \in G \times \widehat{G} .
$$

2. Wilson bases for general lattices - the continuous case. We first show that all lattices in $\mathbb{R}^{2}$ which are important for applications, such as the rectangular lattice, the hexagonal lattice, and lattices whose generator matrix has rational entries, possess a uniquely determined matrix in Hermite normal form, which we will call the canonical generator matrix. In particular, we characterize exactly those lattices, which possess a generator matrix in Hermite normal form.

We then show that it is possible to construct orthonormal Wilson bases for timefrequency lattices $\Lambda$ with $\operatorname{vol}(\Lambda)=\frac{1}{2}$, which possess a generator matrix in Hermite normal form. In principle Wilson systems can be defined for lattices $\Lambda$ with $\operatorname{vol}(\Lambda) \neq$ $\frac{1}{2}$, however so far all known constructions of orthogonal Wilson bases for $L^{2}(\mathbb{R})$ are strictly tied to lattices with volume $\frac{1}{2}$. In light of this fact throughout this paper a Wilson system will always be associated with a time-frequency lattice of volume $\frac{1}{2}$.

LEMma 2.1. Let $\Lambda$ be a lattice in $\mathbb{R}^{2}$. Then the following conditions are equivalent.

(i) $P_{2}(\Lambda)$ is discrete, where $P_{2}: \mathbb{R}^{2} \rightarrow \mathbb{R},(x, y) \mapsto y$.

(ii) There exists a generator matrix $A$ for $\Lambda$ which is in Hermite normal form. If one of these conditions is satisfied, the matrix $A$ is uniquely determined.

Proof. Let

$$
A^{\prime}=\left[\begin{array}{ll}
a^{\prime} & b^{\prime} \\
c^{\prime} & d^{\prime}
\end{array}\right]
$$

be an arbitrary generator matrix for $\Lambda$.

First we prove that (ii) implies (i). By (ii), there exists a matrix

$$
A=\left[\begin{array}{ll}
a & b \\
0 & d
\end{array}\right]
$$

which is in Hermite normal form and which satisfies $A \mathbb{Z}^{2}=\Lambda$. Thus $P_{2}(\Lambda)=d \mathbb{Z}$, which yields (i).

Next we show (i) $\Rightarrow$ (ii). For this, we construct a matrix

$$
A=\left[\begin{array}{ll}
a & b \\
0 & d
\end{array}\right]
$$


which satisfies the claimed properties. Without loss of generality we assume that $d^{\prime} \neq 0$ (if $d^{\prime}=0$ and $c^{\prime} \neq 0$ we could change the columns of $A^{\prime}$ ). We begin with the following observation. Assume that $\frac{c^{\prime}}{d^{\prime}}$ is not rational. Since $P_{2}(\Lambda)$ is a non-trivial discrete, additive subgroup of $\mathbb{R}$, hence a lattice, there exists $s \in \mathbb{R} \backslash\{0\}$ such that $P_{2}(\Lambda)=s \mathbb{Z}$. Thus $c^{\prime}=s m$ and $d^{\prime}=s n$ for some $m, n \in \mathbb{Z}$, a contradiction. This implies that the quotient $\frac{c^{\prime}}{d^{\prime}}$ is rational. Setting $\frac{c^{\prime}}{d^{\prime}}=\frac{k}{l}, k, l \in \mathbb{Z}$ with $\operatorname{gcd}(k, l)=1$ and factoring out $\frac{d^{\prime}}{l}$, without loss of generality we can assume that $A^{\prime}$ is of the form

$$
A^{\prime}=r\left[\begin{array}{ll}
a^{\prime} & b^{\prime} \\
c^{\prime} & d^{\prime}
\end{array}\right]
$$

with $a^{\prime}, b^{\prime}, r \in \mathbb{R}$ and $c^{\prime}, d^{\prime} \in \mathbb{Z}$. Now we proceed as follows. First we set $p:=$ $\operatorname{gcd}\left(c^{\prime}, d^{\prime}\right)>0$. We then obtain

$$
A^{\prime}\left[\begin{array}{l} 
\pm \frac{d^{\prime}}{p} \\
\mp \frac{c^{\prime}}{p}
\end{array}\right]=\left[\begin{array}{c} 
\pm \frac{r \operatorname{det}\left(A^{\prime}\right)}{p} \\
0
\end{array}\right] \in \Lambda,
$$

since $\frac{c^{\prime}}{p}, \frac{d^{\prime}}{p} \in \mathbb{Z}$. Hence we can define $a$ by $a:=\left|\frac{r \operatorname{det}\left(A^{\prime}\right)}{p}\right|>0$.

In a second step we compute $b$ and $d$. Since $\frac{c^{\prime}}{p}$ and $\frac{d^{\prime}}{p}$ are relative prime, there exist $m, n \in \mathbb{Z}$ such that $\frac{c^{\prime}}{p} m+\frac{d^{\prime}}{p} n=1$ (see [24] Theorem 4.4]). Hence $c^{\prime} m+d^{\prime} n=p$ and we obtain

$$
A^{\prime}\left[\begin{array}{c}
m \\
n
\end{array}\right]=\left[\begin{array}{c}
r\left(a^{\prime} m+b^{\prime} n\right) \\
r p
\end{array}\right] \in \Lambda .
$$

Now let $k \in \mathbb{Z}$ be chosen in such a way that $0 \leq r\left(a^{\prime} m+b^{\prime} n\right)+k a<a$ and define $b:=k a+r\left(a^{\prime} m+b^{\prime} n\right)$ and $d:=r p$. Without loss of generality we can assume that $d>0$, since otherwise we just take $-m$ and $-n$ instead of $m$ and $n$. Then

$$
\left[\begin{array}{l}
b \\
d
\end{array}\right]=\left[\begin{array}{c}
r\left(a^{\prime} m+b^{\prime} n\right) \\
r p
\end{array}\right]+k\left[\begin{array}{l}
a \\
0
\end{array}\right] \in \Lambda
$$

and $|a d|=\left|\frac{r \operatorname{det}\left(A^{\prime}\right)}{p}\right| r p=\left|r^{2} \operatorname{det}\left(A^{\prime}\right)\right|$. This proves that $A$ generates $\Lambda$ and is in Hermite normal form.

At last we prove that the matrix in condition (ii) is uniquely determined. For this, assume there exist $a, b, d, a^{\prime}, b^{\prime}, d^{\prime} \in \mathbb{R}$ with $a, a^{\prime}, d, d^{\prime}>0,0 \leq b<a$ and $0 \leq b^{\prime}<a^{\prime}$ such that

$$
\Lambda=A \mathbb{Z}^{2}=A^{\prime} \mathbb{Z}^{2}
$$

where

$$
A=\left[\begin{array}{ll}
a & b \\
0 & d
\end{array}\right] \quad \text { and } \quad A^{\prime}=\left[\begin{array}{cc}
a^{\prime} & b^{\prime} \\
0 & d^{\prime}
\end{array}\right]
$$

By (2.1), there exist $m, n \in \mathbb{Z}$ with $a^{\prime}=m a+n b$ and $0=n d$, which implies that $a^{\prime}=m a$. Again by (2.1), we can find $k, l \in \mathbb{Z}$ such that $b^{\prime}=k a+l b$ and $d^{\prime}=l d$. Since $\operatorname{vol}(\Lambda)=|a d|=\left|a^{\prime} d^{\prime}\right|$, we obtain $|m l|=1$. Now $a, a^{\prime}, d, d^{\prime}>0$ implies that $a=a^{\prime}$, $d=d^{\prime}$, and $l=1$. Finally, applying this to $b^{\prime}=k a+l b$ and using that $0 \leq b<a$ and $0 \leq b^{\prime}<a^{\prime}$ yields $b=b^{\prime}$. Thus we have shown $A=A^{\prime}$, which completes the proof. 
In the following we restrict our attention to lattices, which possess a generator matrix in Hermite normal form. All results in the situation $L^{2}(\mathbb{R})$ could be derived (in the same manner, but with much more technical effort) for general lattices, however with little or no practical benefit.

Definition 2.2. Let $\Lambda$ be a lattice in $\mathbb{R}^{2}$, which satisfies the conditions of Lemma 2.1. Then the uniquely determined generator matrix A of Lemma 2.1 is called the canonical generator matrix for $\Lambda$.

Now let $\Lambda$ be a lattice with $\operatorname{vol}(\Lambda)=\frac{1}{2}$, which possesses a generator matrix in Hermite normal form. Using the definition of a canonical generator matrix, we define a Wilson system associated with $\Lambda$ as follows.

DeFinition 2.3. If $\mathcal{G}(g, \Lambda) \subseteq L^{2}(\mathbb{R})$ is a Gabor system of redundancy 2, and

$$
A=\left[\begin{array}{ll}
a & b \\
0 & d
\end{array}\right]
$$

is the canonical generator matrix for the lattice $\Lambda$, then the associated Wilson system $\mathcal{W}\left(g, \Lambda, L^{2}(\mathbb{R})\right)=\left\{\psi_{m, n}^{\Lambda}\right\}_{m \in \mathbb{Z}, n \geq 0}$ consists of the functions

$$
\begin{array}{ll}
\psi_{m, 0}^{\Lambda}=g_{2 m a, 0}, & \text { if } n=0, \\
\psi_{m, n}^{\Lambda}=\frac{1}{\sqrt{2}} e^{-\pi i b d n^{2}}\left(g_{m a+n b, n d}+g_{m a-n b,-n d}\right), & \text { if } n \neq 0, m+n \text { even }, \\
\psi_{m, n}^{\Lambda}=\frac{i}{\sqrt{2}} e^{-\pi i b d n^{2}}\left(g_{m a+n b, n d}-g_{m a-n b,-n d}\right), & \text { if } n \neq 0, m+n \text { odd. }
\end{array}
$$

If the system $\mathcal{W}\left(g, \Lambda, L^{2}(\mathbb{R})\right)$ is an orthonormal basis for $L^{2}(\mathbb{R})$ we call it a Wilson (orthonormal) basis.

We will see that this definition reduces to the usual definition of Wilson systems. For this, we consider the rectangular lattice

$$
\Gamma=\left\{\left(\frac{m}{2}, n\right)\right\}_{m, n \in \mathbb{Z}}
$$

It is an easy calculation to show that the canonical generator matrix for $\Gamma$ is

$$
A=\left[\begin{array}{cc}
\frac{1}{2} & 0 \\
0 & 1
\end{array}\right]
$$

Thus, for each $g \in L^{2}(\mathbb{R})$, the Wilson system $\mathcal{W}\left(g, \Gamma, L^{2}(\mathbb{R})\right)$ consists indeed of the functions

$$
\begin{array}{ll}
\psi_{m, 0}^{\Gamma}=g_{m, 0}, & \text { if } n=0, \\
\psi_{m, n}^{\Gamma}=\frac{1}{\sqrt{2}}\left(g_{m / 2, n}+g_{m / 2,-n}\right), & \text { if } m+n \text { is even, } \\
\psi_{m, n}^{\Gamma}=\frac{i}{\sqrt{2}}\left(g_{m / 2, n}-g_{m / 2,-n}\right), & \text { if } m+n \text { is odd, }
\end{array}
$$

which coincides with the usual definition of Wilson systems, cf. for instance [20 Definition 8.5.1]. Notice that we will fix the notation $\Gamma$ for the remainder.

We will make use of the following well-known theorem about a Wilson system for rectangular lattices to constitute an orthonormal basis (e.g. cf. [3] Theorem 4.1]).

Theorem 2.4. Suppose that $g \in L^{2}(\mathbb{R})$ is such that

(a) $\hat{g}$ is real-valued and

(b) $\left\{g_{m / 2, n}\right\}_{m, n \in \mathbb{Z}}$ is a tight Gabor frame for $L^{2}(\mathbb{R})$ with frame bound 2 . 
Then the system $\mathcal{W}\left(g, \Gamma, L^{2}(\mathbb{R})\right)$ is a Wilson orthonormal basis for $L^{2}(\mathbb{R})$.

We are now ready to extend the construction of Wilson bases for time-frequency lattices which possess a generator matrix in Hermite normal form.

TheOREM 2.5. Let $\Lambda$ be a lattice in $\mathbb{R}^{2}$ with $\operatorname{vol}(\Lambda)=\frac{1}{2}$ and canonical generator matrix

$$
A=\left[\begin{array}{ll}
a & b \\
0 & d
\end{array}\right]
$$

Define $U$ by

$$
U:=\mathcal{D}_{1 / d} \circ \mathcal{F} \circ \mathcal{N}_{-b / d} \circ \mathcal{F}^{-1} .
$$

Let $g \in L^{2}(\mathbb{R})$ be such that

(i) $\widehat{U g}$ is real-valued,

(ii) $\left\{g_{m a+n b, n d}\right\}_{m, n \in \mathbb{Z}}$ is a tight frame for $L^{2}(\mathbb{R})$ with frame bound 2 .

Then the system $\mathcal{W}\left(g, \Lambda, L^{2}(\mathbb{R})\right)$ is a Wilson orthonormal basis for $L^{2}(\mathbb{R})$.

Proof. We will reduce our claim to Theorem 2.4 by using a metaplectic transform. We define $\mathcal{A}$ by

$$
\mathcal{A}=\left[\begin{array}{cc}
d & -b \\
0 & 2 a
\end{array}\right]
$$

Then, for all $m, n \in \mathbb{Z}$, we have

$$
\mathcal{A}(m a+n b, n d)=\left(\frac{1}{2} m, n\right) .
$$

Since $\mathcal{A} \in \operatorname{Sp}(2, \mathbb{R})$ we can apply [17, Theorem 4.51] and write

$$
\mathcal{A}=B_{d}(-\mathcal{J}) C_{b / d} \mathcal{J} .
$$

By [20, Example 9.4.1] we obtain

$$
\rho(x, y, 1) g=U^{-1} \rho(\mathcal{A}(x, y), 1)(U g),
$$

with $U$ defined in (2.2). Using (1.1), (2.3), and (2.4), we compute

$$
\begin{aligned}
g_{m a+n b, n d} & =e^{\pi i(m a+n b) n d} \rho(m a+n b, n d, 1) g \\
& =e^{\pi i(m a+n b) n d} U^{-1} \rho(\mathcal{A}(m a+n b, n d), 1)(U g) \\
& =e^{\pi i(m a+n b) n d} U^{-1} \rho\left(\frac{1}{2} m, n, 1\right)(U g) .
\end{aligned}
$$

Using (1.1) again, we obtain

$$
g_{m a+n b, n d}=e^{\pi i(m a+n b) n d} e^{-\pi i \frac{1}{2} m n} U^{-1}(U g)_{m / 2, n}=e^{\pi i b d n^{2}} U^{-1}(U g)_{m / 2, n} .
$$

Since multiplication by a phase factor and applying a unitary operator to a tight frame preserves tightness (and frame bounds), it follows that condition (ii) is equivalent to $\left\{(U g)_{m / 2, n}\right\}_{m, n \in \mathbb{Z}}$ being a tight frame with frame bound 2. We need not deal with condition (i), since this states already that $\widehat{U g}$ is real-valued. Applying Theorem 2.4 yields that the Wilson system $\mathcal{W}\left(U g, \Gamma, L^{2}(\mathbb{R})\right)$ is an orthonormal basis. Using now the metaplectic transform, i.e., (2.5), and the fact that $U$ is a unitary operator, finishes the proof. 
We will conclude this section by providing an example, which shows how we can compute a Wilson basis with excellent time-frequency localization for a special lattice, but this calculation can also be done for an arbitrary lattice.

Here we consider the hexagonal lattice $\Lambda_{H}$ with generator matrix

$$
A_{H}=\left[\begin{array}{cc}
\frac{\sqrt{2}}{\sqrt[4]{3}} & \frac{\sqrt{2}}{2 \sqrt[4]{3}} \\
0 & \frac{\sqrt[4]{3}}{\sqrt{2}}
\end{array}\right]
$$

This lattice was also used in 33 . Observe that since we have $0 \leq \frac{\sqrt{2}}{2 \sqrt[4]{3}}<\frac{\sqrt{2}}{\sqrt[4]{3}}$, the matrix $A_{H}$ is already the canonical generator matrix for $\Lambda_{H}$. First we define the function $h \in L^{2}(\mathbb{R})$ by

$$
h(x)=(2 \nu)^{\frac{1}{4}} e^{-\nu \pi x^{2}} .
$$

By [20] Theorem 7.5.3], the set $\left\{h_{m / 2, n}\right\}_{m, n \in \mathbb{Z}}$ is a frame for $L^{2}(\mathbb{R})$. Let $S$ denote its frame operator and consider the function $\varphi \in L^{2}(\mathbb{R})$ given by

$$
\varphi=\sqrt{2} \mathcal{F} \circ S^{-\frac{1}{2}} h .
$$

Using [3, Theorem 4.6], this function coincides with the function considered in [1] Section 4]. There it was shown that $\varphi$ satisfies conditions (a) and (b) of Theorem 2.4 and hence yields a Wilson basis in the sense of Theorem 2.4 Moreover, $\varphi$ has exponential decay in time and frequency. To obtain a generating function for a Wilson basis with respect to $\Lambda_{H}$ in the sense of Theorem 2.5 first observe that, by the proof of Theorem 2.5. we only need to compute the function $g=U^{-1} \varphi$, where $U$ is defined in (2.2). Then $g$ automatically satisfies conditions (i) and (ii) of Theorem 2.5] and hence the system $\mathcal{W}\left(g, A_{H}, L^{2}(\mathbb{R})\right)$ is a Wilson orthonormal basis by Theorem [2.5 Thus we define $g \in L^{2}(\mathbb{R})$ by

$$
g=\mathcal{F} \circ \mathcal{N}_{\frac{1}{\sqrt{3}}} \circ \mathcal{F}^{-1} \circ \mathcal{D}_{\frac{\sqrt[4]{3}}{\sqrt{2}}} \varphi=\sqrt{2} \mathcal{F} \circ \mathcal{N}_{\frac{1}{\sqrt{3}}} \circ \mathcal{D}_{\frac{\sqrt{2}}{\sqrt[4]{3}}} \circ S^{-\frac{1}{2}} h
$$

Let us mention that the function $g$ has exponential decay in time and frequency. Thus we obtain a Wilson basis with respect to the lattice $\Lambda$ with very good timefrequency localization. As already mentioned above this procedure can be applied to an arbitrary lattice, hence we obtain a Wilson basis with excellent time-frequency localization for any lattice.

3. Wilson bases for general lattices - the discrete case. In this section we analyze the construction of Wilson bases for general time-frequency lattices for functions defined on $\ell^{2}(\mathbb{Z})$. The reasons for considering the setting $\ell^{2}(\mathbb{Z})$ are on that the one hand several applications such as filter bank design in digital signal processing deal directly with a discrete setting [7, and on the other hand, even those problems that arise in the "continuous" setting of $L^{2}(\mathbb{R})$ require a discrete model for their numerical treatment. Thus, with these practical aspects in mind, throughout this section we naturally consider only lattices whose generator matrices have rational entries, since any implementation is intrinsically restricted to such "rationally" generated lattices. Another natural setting for numerical implementations is of course $\mathbb{C}^{L}$ (which can be identified with the space of $L$-periodic sequences). We will analyze that case in the next section. 
Before we proceed we define Gabor systems and Wilson systems on $\ell^{2}(\mathbb{Z})$ for general time-frequency lattices $\Lambda$ with $\operatorname{vol}(\Lambda)=\frac{1}{2}$. First we prove that each lattice possesses a generator matrix of some particular form.

Lemma 3.1. Let $\Lambda$ be a lattice in $\mathbb{Z} \times \mathbb{R}$ with generator matrix $A$ given by

$$
A=\left[\begin{array}{ll}
a & b \\
c & d
\end{array}\right], \quad \text { with } a, b \in \mathbb{Z}, c, d \in \mathbb{Q} \text {, and } \operatorname{det}(A)=\frac{1}{2},
$$

and denote $c=\frac{p}{q}, d=\frac{p^{\prime}}{q^{\prime}}$ with $\operatorname{gcd}(p, q)=\operatorname{gcd}\left(p^{\prime}, q^{\prime}\right)=1, p, q, p^{\prime}, q^{\prime} \in \mathbb{Z}$. Then $\Lambda$ possesses a uniquely determined generator matrix of the form

$$
A^{\prime}=\left[\begin{array}{cc}
\frac{N}{2} & b^{\prime} \\
0 & \frac{1}{N}
\end{array}\right]
$$

where $N=\frac{q q^{\prime}}{\operatorname{gcd}\left(p q^{\prime}, p^{\prime} q\right)}$ and $b^{\prime} \in \mathbb{Z}, 0 \leq b^{\prime}<\frac{N}{2}$.

Proof. We assume that $c \neq 0$, otherwise (3.1) is automatically satisfied. We first show that $A$ can be written as

$$
A=\left[\begin{array}{cc}
a & b \\
\frac{r}{N} & \frac{s}{N}
\end{array}\right],
$$

with integers $r, s, N$, such that $\operatorname{gcd}(r, s)=1$.

Let $c=\frac{p}{q}, d=\frac{p^{\prime}}{q^{\prime}}$ with $p, p^{\prime}, q, q^{\prime} \in \mathbb{Z}$, denote $N^{\prime}:=q q^{\prime}, \tilde{c}:=p q^{\prime}, \tilde{d}:=p^{\prime} q$ and write $z=\operatorname{gcd}(\tilde{c}, \tilde{d})$. Since $a, b, \tilde{c}, \tilde{d} \in \mathbb{Z}$ and since

$$
\operatorname{vol}(\Lambda)=\frac{1}{2} \Rightarrow a \tilde{d}-b \tilde{c}=\frac{N^{\prime}}{2}
$$

it follows that $\frac{N^{\prime}}{2} \in \mathbb{Z}$. A necessary and sufficient condition for the equation $a \tilde{d}-b \tilde{c}=$ $\frac{N^{\prime}}{2}$ to have an integer solution in $a$ and $b$ is that $\operatorname{gcd}(\tilde{c}, \tilde{d}) \mid \frac{N^{\prime}}{2}$, see [24] Theorem 8.1], hence $z \mid \frac{N^{\prime}}{2}$. Denote $z^{\prime}:=\frac{N^{\prime}}{2 z}, r:=\frac{\tilde{c}}{z}, s:=\frac{\tilde{d}}{z}$. Then $c=\frac{r}{2 z^{\prime}}, d=\frac{s}{2 z^{\prime}}$ with $z^{\prime} \in \mathbb{Z}$ and $\operatorname{gcd}(r, s)=1$. By a proper choice of the signs of $c$ and $d$ we can always assume that $z^{\prime}$ is positive. By writing $N:=2 z^{\prime} \in \mathbb{Z}$ we see that $A$ can indeed be written as in (3.2).

Now, assuming that $A$ is of the form (3.2), we compute

$$
\left[\begin{array}{ll}
a & b \\
c & d
\end{array}\right]\left[\begin{array}{c}
N d \\
-N c
\end{array}\right]=\left[\begin{array}{c}
N(a d-b c) \\
0
\end{array}\right]=\left[\begin{array}{c}
\frac{N}{2} \\
0
\end{array}\right] .
$$

Since $\operatorname{gcd}(r, s)=1$ there exist integers $m, n$ with $m r+n s=1$. For such a pair $(m, n)$ we denote $b^{\prime}=a m+b n$ and obtain

$$
\left[\begin{array}{ll}
a & b \\
c & d
\end{array}\right]\left[\begin{array}{c}
m \\
n
\end{array}\right]=\left[\begin{array}{c}
a m+b n \\
\frac{r}{N} m+\frac{s}{N} n
\end{array}\right]=\left[\begin{array}{c}
b^{\prime} \\
\frac{1}{N}
\end{array}\right] .
$$

If $b^{\prime}<0$ or $b^{\prime} \geq \frac{N}{2}$, we substitute the vector obtained by

$$
\left[\begin{array}{c}
b^{\prime} \\
\frac{1}{N}
\end{array}\right]+k\left[\begin{array}{c}
\frac{N}{2} \\
0
\end{array}\right]=\left[\begin{array}{c}
b^{\prime}+k \frac{N}{2} \\
\frac{1}{N}
\end{array}\right]
$$

where $k \in \mathbb{Z}$ is chosen in such a way that $0 \leq b^{\prime}+k \frac{N}{2}<\frac{N}{2}$. Consequently the matrix

$$
\left[\begin{array}{cc}
\frac{N}{2} & b^{\prime} \\
0 & \frac{1}{N}
\end{array}\right]
$$


generates the lattice $\Lambda$. Finally, since $\frac{N}{2}$ and $b^{\prime}$ are integers and $0 \leq b^{\prime}<\frac{N}{2}$, the generator matrix in (3.3) is indeed of the form (3.1).

The fact that this is a unique representation follows immediately from the condition $0 \leq b^{\prime}<\frac{N}{2}$.

DeFINITION 3.2. Let $\Lambda$ be a lattice in $\mathbb{Z} \times \mathbb{R}$. Then the uniquely determined matrix $A^{\prime}$ of Lemma 3.1 is called the canonical generator matrix for $\Lambda$.

In the following we will regard such a lattice as a lattice in $\mathbb{Z} \times \mathbb{T}$ by considering $\Lambda=\left\{\frac{N}{2} m+b n, e^{2 \pi i \frac{n}{N}}\right\}_{m \in \mathbb{Z}, n=0, \ldots, N-1}$. This is a very natural approach, since, for all $k \in \mathbb{Z}$, we have

$$
\left(\frac{N}{2} m+b n, \frac{1}{N} n+k\right)=\left(\frac{N}{2}(m-2 b k)+b(n+k N), \frac{1}{N}(n+k N)\right),
$$

hence the lattice $A^{\prime} \mathbb{Z}^{2}$ is invariant under adding integers to the second component. Moreover, it is sufficient to restrict to the index set $\mathbb{Z} \times\{0, \ldots, N-1\}$, since, for all $0 \leq n^{\prime}<N$ and $k \in \mathbb{Z}$,

$$
\left(\frac{N}{2} m+b\left(n^{\prime}+k N\right),\left(n^{\prime}+k N\right) \bmod N\right)=\left(\frac{N}{2}(m+2 b k)+b n^{\prime}, n^{\prime}\right),
$$

which implies

$$
\left\{\left(\frac{N}{2} m+b n, n \bmod N\right)\right\}_{m, n \in \mathbb{Z}}=\left\{\left(\frac{N}{2} m+b n, n\right)\right\}_{m \in \mathbb{Z}, n=0, \ldots, N-1}
$$

in the sense of sets.

Using the definition of canonical generator matrices we can now define Gabor systems for $\ell^{2}(\mathbb{Z})$.

DeFINITION 3.3. Let $\Lambda$ be a lattice in $\mathbb{Z} \times \mathbb{T}$ with canonical generator matrix $A$ given by

$$
A=\left[\begin{array}{cc}
\frac{N}{2} & b \\
0 & \frac{1}{N}
\end{array}\right]
$$

and let $g \in \ell_{2}(\mathbb{Z})$. Then the associated Gabor system $\left\{g_{m \frac{N}{2}+n b, n \frac{1}{N}}\right\}_{m \in \mathbb{Z}, n=0, \ldots, N-1}$ is given by

$$
g_{m \frac{N}{2}+n b, n \frac{1}{N}}(l)=g\left(l-\left(m \frac{N}{2}+n b\right)\right) e^{2 \pi i l n / N}, \quad l \in \mathbb{Z} .
$$

Now we first give the definition of a Wilson basis associated with a lattice with diagonal canonical generator matrix, i.e., with $b=0$ (in this special case the definition coincides with the one given in [6]).

Definition 3.4. Let $\Lambda$ be a lattice in $\mathbb{Z} \times \mathbb{T}$ with canonical generator matrix $A$ given by

$$
A=\left[\begin{array}{cc}
\frac{N}{2} & 0 \\
0 & \frac{1}{N}
\end{array}\right]
$$

and let $g \in \ell_{2}(\mathbb{Z})$. Then the Wilson system $\mathcal{W}\left(g, \Lambda, \ell_{2}(\mathbb{Z})\right)=\left\{\psi_{m, n}\right\}_{m \in \mathbb{Z}, n=0, \ldots, \frac{N}{2}}$ is given by

$$
\psi_{m, n}^{\Lambda}=g_{m N, n \frac{1}{N}}, \quad \text { if } m \in \mathbb{Z}, n=0, \frac{N}{2},
$$

and for $m \in \mathbb{Z}, n=1, \ldots, \frac{N}{2}-1$,

$$
\begin{array}{ll}
\psi_{m, n}^{\Lambda}=\frac{1}{\sqrt{2}}\left(g_{m \frac{N}{2}, n \frac{1}{N}}+g_{\left.m \frac{N}{2},-n \frac{1}{N}\right),} \text { if } m+n \text { even },\right. \\
\psi_{m, n}^{\Lambda}=\frac{i}{\sqrt{2}}\left(g_{m \frac{N}{2}, n \frac{1}{N}}-g_{m \frac{N}{2},-n \frac{1}{N}}\right), & \text { if } m+n \text { odd. }
\end{array}
$$


The Zak transform, which can be defined for any locally compact abelian group (cf. 28]), will be employed to prove equivalent conditions for the Wilson system to form an orthonormal basis. In particular, we need the Zak transform on $\mathbb{T}$ with respect to the uniform lattice $K=\left\{e^{2 \pi i \frac{2 k}{N}}: k=0, \ldots, \frac{N}{2}-1\right\}$ in $\mathbb{T}$, which is defined on the set of square-integrable functions on $\left\{e^{2 \pi i t}: t \in\left[0, \frac{2}{N}\right)\right\} \times\left\{0, \ldots, \frac{N}{2}-1\right\}$ by

$$
Z f\left(e^{2 \pi i t}, y\right)=\sum_{k=0}^{\frac{N}{2}-1} f\left(e^{2 \pi i\left(t+\frac{2 k}{N}\right)}\right) e^{2 \pi i \frac{2 k}{N} y}
$$

The proof of the following proposition is inspired by the proof of [11, Proposition $5.2]$.

Proposition 3.5. Let $g \in \ell_{2}(\mathbb{Z})$ be such that $\hat{g}$ is real-valued and consider the lattice $\Lambda$ with canonical generator matrix given by

$$
\left[\begin{array}{ll}
\frac{N}{2} & 0 \\
0 & \frac{1}{N}
\end{array}\right]
$$

Then the following conditions are equivalent.

(i) $\left\{g_{m \frac{N}{2}, n \frac{1}{N}}\right\}_{m \in \mathbb{Z}, n=0, \ldots, N-1}$ is a tight frame for $\ell_{2}(\mathbb{Z})$ with frame bound 2 .

(ii) We have $\left|Z \hat{g}\left(e^{2 \pi i t}, y\right)\right|^{2}+\left|Z \hat{g}\left(e^{2 \pi i\left(t+\frac{1}{N}\right)}, y\right)\right|^{2}=N$ a.e..

(iii) For all $j \in\{0, \ldots, N-1\}$, we have $\sum_{l=0}^{N-1} \hat{g}\left(e^{2 \pi i\left(t+\frac{l}{N}\right)}\right) \hat{g}\left(e^{2 \pi i\left(t+\frac{l+2 j}{N}\right)}\right)=N \delta_{j, 0}$ a.e..

(iv) $\mathcal{W}\left(g, \Lambda, \ell_{2}(\mathbb{Z})\right)$ is an orthonormal basis for $\ell_{2}(\mathbb{Z})$.

Proof. Throughout this proof we choose the normalized Haar measure on $\mathbb{T}$, i.e., $m(E)=\int_{0}^{1} 1_{E}\left(e^{2 \pi i t}\right) d t$ for all measurable $E \subseteq \mathbb{T}$ and the counting measure on its dual group $\widehat{\mathbb{T}}=\mathbb{Z}$. This choice ensures that the Plancherel formula for $\mathbb{T}$ holds.

Since we will mainly work in the Fourier domain, we first need to compute the Fourier transform of the elements of the Gabor system for our following calculations:

$$
\begin{aligned}
\widehat{g_{m \frac{N}{2}, n \frac{1}{N}}}\left(e^{2 \pi i t}\right) & =\sum_{l \in \mathbb{Z}} e^{2 \pi i \frac{n}{N} l} g\left(e^{2 \pi i\left(l-m \frac{N}{2}\right)}\right) e^{-2 \pi i l t} \\
& =e^{2 \pi i \frac{m n}{2}} e^{-2 \pi i t m \frac{N}{2}} \sum_{l \in \mathbb{Z}} g\left(e^{2 \pi i l}\right) e^{-2 \pi i l\left(t-\frac{n}{N}\right)} \\
& =(-1)^{m n} \hat{g}_{n \frac{1}{N},-m \frac{N}{2}}\left(e^{2 \pi i t}\right) .
\end{aligned}
$$

(i) $\Leftrightarrow$ (ii): Since the Fourier transform is a unitary operator, the Gabor system $\left\{g_{m \frac{N}{2}, \frac{1}{N} n}\right\}_{m \in \mathbb{Z}, n=0, \ldots, N-1}$ is a tight frame for $\ell_{2}(\mathbb{Z})$ with frame bound 2 if and only if the Gabor system $\left\{\hat{g}_{n \frac{1}{N}, m \frac{N}{2}}\right\}_{m \in \mathbb{Z}, n=0, \ldots, N-1}$ is a tight frame for $L^{2}(\mathbb{T})$ with frame bound 2. Then we write this set as the disjoint union $\left\{\hat{g}_{n \frac{2}{N}, m \frac{N}{2}}\right\}_{m \in \mathbb{Z}, n=0, \ldots, \frac{N}{2}-1} \cup$ $\left\{\left(T_{-\frac{1}{N}} \hat{g}\right)_{n \frac{2}{N}, m \frac{N}{2}}\right\}_{m \in \mathbb{Z}, n=0, \ldots, \frac{N}{2}-1}=: G_{1} \cup G_{2}$, and let $S_{i}$ denote the frame operator for $G_{i}, i \stackrel{N}{=} 1,2$.

First we compute the frame operator $S_{1}$. For all $f \in L^{2}(\mathbb{T})$, using the Poisson summation formula [18. Theorem 4.42] applied to $H=\left\{e^{2 \pi i k \frac{2}{N}}: k=0, \ldots, \frac{N}{2}-1\right\}$, we obtain

$$
S_{1} f\left(e^{2 \pi i t}\right)=\sum_{m \in \mathbb{Z}} \sum_{n=0}^{\frac{N}{2}-1}\left\langle f, \hat{g}_{n \frac{2}{N}, m \frac{N}{2}}\right\rangle \hat{g}_{n \frac{2}{N}, m \frac{N}{2}}\left(e^{2 \pi i t}\right)
$$




$$
\begin{aligned}
& =\sum_{m \in \mathbb{Z}} \sum_{n=0}^{\frac{N}{2}-1} \int_{0}^{1} f\left(e^{2 \pi i s}\right) \overline{\hat{g}\left(e^{2 \pi i\left(s-n \frac{2}{N}\right)}\right)} e^{-2 \pi i m \frac{N}{2} s} d s \hat{g}\left(e^{2 \pi i\left(t-n \frac{2}{N}\right)}\right) e^{2 \pi i m \frac{N}{2} t} \\
& =\sum_{n=0}^{\frac{N}{2}-1}\left[\sum_{m \in \mathbb{Z}}\left(\widehat{f} \overline{\overline{T_{n \frac{2}{N}} \hat{g}}}\right)\left(m \frac{N}{2}\right) e^{2 \pi i m \frac{N}{2} t}\right] \hat{g}\left(e^{2 \pi i\left(t-n \frac{2}{N}\right)}\right) \\
& =\sum_{n=0}^{\frac{N}{2}-1} \frac{2}{N} \sum_{k=0}^{\frac{N}{2}-1}\left(f \overline{T_{n \frac{2}{N}} \hat{g}}\right)\left(e^{2 \pi i\left(t+k \frac{2}{N}\right)}\right) \hat{g}\left(e^{2 \pi i\left(t-n \frac{2}{N}\right)}\right) .
\end{aligned}
$$

Applying now the Zak transform yields

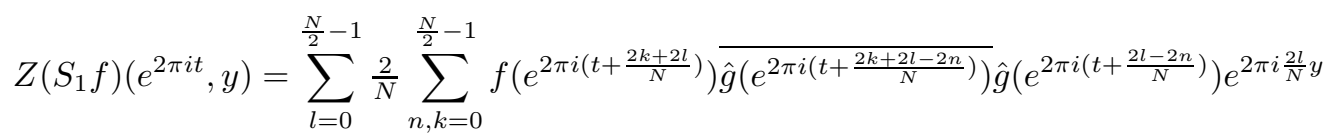

$$
\begin{aligned}
& =\frac{2}{N} \sum_{l, n, k=0}^{\frac{N}{2}-1} f\left(e^{2 \pi i\left(t+\frac{2 l+2 n}{N}\right)}\right) \overline{\hat{g}\left(e^{2 \pi i\left(t+\frac{2 l}{N}\right)}\right)} \hat{g}\left(e^{2 \pi i\left(t+\frac{2 l-2 k}{N}\right)}\right) e^{2 \pi i \frac{2(l-k+n)}{N} y} \\
& =\frac{2}{N} \sum_{l=0}^{\frac{N}{2}-1} \overline{\hat{g}\left(e^{2 \pi i\left(t+\frac{2 l}{N}\right)}\right)}\left[\sum_{k=0}^{\frac{N}{2}-1} \hat{g}\left(e^{2 \pi i\left(t+\frac{2 l-2 k}{N}\right)}\right) e^{-2 \pi i \frac{2 k}{N} y}\right] \\
& \cdot\left[\sum_{n=0}^{\frac{N}{2}-1} f\left(e^{2 \pi i\left(t+\frac{2 n+2 l}{N}\right)}\right) e^{2 \pi i \frac{2 n}{N} y}\right] e^{2 \pi i \frac{2 l}{N} y} \\
& =\frac{2}{N} \overline{Z(\hat{g})\left(e^{2 \pi i t}, y\right)} Z(\hat{g})\left(e^{2 \pi i t}, y\right) Z(f)\left(e^{2 \pi i t}, y\right) .
\end{aligned}
$$

To compute the Zak transform of $S_{2}$, we can use the previous calculation, which yields

$$
\begin{aligned}
Z\left(S_{2} f\right)\left(e^{2 \pi i t}, y\right) & =\frac{2}{N} Z(f)\left(e^{2 \pi i t}, y\right)\left|Z\left(T_{-\frac{1}{N}} \hat{g}\right)\left(e^{2 \pi i t}, y\right)\right|^{2} \\
& =\frac{2}{N} Z(f)\left(e^{2 \pi i t}, y\right)\left|Z(\hat{g})\left(e^{2 \pi i\left(t+\frac{1}{N}\right)}, y\right)\right|^{2}
\end{aligned}
$$

since

$$
Z\left(T_{-\frac{1}{N}} \hat{g}\right)\left(e^{2 \pi i t}, y\right)=\sum_{k=0}^{\frac{N}{2}-1} \hat{g}\left(e^{2 \pi i\left(t+\frac{2 k}{N}+\frac{1}{N}\right)}\right) e^{2 \pi i \frac{2 k}{N} y}=Z(\hat{g})\left(e^{2 \pi i\left(t+\frac{1}{N}\right)}, y\right) .
$$

Hence (i) is equivalent to

$$
\begin{aligned}
2 Z(f)\left(e^{2 \pi i t}, y\right) & =Z\left(\left(S_{1}+S_{2}\right) f\right)\left(e^{2 \pi i t}, y\right) \\
& =\frac{2}{N} Z(f)\left(e^{2 \pi i t}, y\right)\left[\left|Z(\hat{g})\left(e^{2 \pi i t}, y\right)\right|^{2}+\left|Z(\hat{g})\left(e^{2 \pi i\left(t+\frac{1}{N}\right)}, y\right)\right|^{2}\right] \text { a.e. }
\end{aligned}
$$

which holds if and only if (ii) is satisfied.

(ii) $\Leftrightarrow$ (iii): The following properties of the Zak transform will be exploit several times. The reconstruction formula

$$
\sum_{y=0}^{\frac{N}{2}-1} Z f\left(e^{2 \pi i t}, y\right)=\sum_{k=0}^{\frac{N}{2}-1} f\left(e^{2 \pi i\left(t+\frac{2 k}{N}\right)}\right) \sum_{y=0}^{\frac{N}{2}-1} e^{2 \pi i \frac{2 k}{N} y}=\frac{N}{2} f\left(e^{2 \pi i t}\right)
$$


holds a.e., since $\sum_{y=0}^{\frac{N}{2}-1} e^{2 \pi i \frac{2 k}{N} y} \neq 0$ if and only if $k=0$ by [23. Lemma 23.29] and, if $k=0$, then $\sum_{y=0}^{\frac{N}{2}-1} e^{2 \pi i \frac{2 k}{N} y}=\frac{N}{2}$. Moreover, we will use that

$$
Z \hat{g}\left(e^{2 \pi i\left(t+\frac{2 l}{N}\right)}, y\right)=\sum_{k=0}^{\frac{N}{2}-1} f\left(e^{2 \pi i\left(t+\frac{2 k+2 l}{N}\right)}\right) e^{2 \pi i \frac{2 k}{N} y}=e^{-2 \pi i \frac{2 l}{N} y} Z \hat{g}\left(e^{2 \pi i t}, y\right) .
$$

The idea is to write the equation in (iii) in terms of the Zak transform. Using the fact that $\hat{g}$ is real-valued, we compute

$$
\begin{aligned}
\sum_{l=0}^{N-1} \hat{g}\left(e^{2 \pi i\left(t+\frac{l}{N}\right)}\right) \hat{g}\left(e^{2 \pi i\left(t+\frac{l+2 j}{N}\right)}\right) & \\
= & \frac{4}{N^{2}} \sum_{l=0}^{N-1} \sum_{x=0}^{\frac{N}{2}-1} Z \hat{g}\left(e^{2 \pi i\left(t+\frac{l}{N}\right)}, x\right) \sum_{y=0}^{\frac{N}{2}-1} \overline{Z \hat{g}\left(e^{2 \pi i\left(t+\frac{l+2 j}{N}\right)}, y\right)} \\
= & \frac{4}{N^{2}} \sum_{k, x, y=0}^{\frac{N}{2}-1}\left[Z \hat{g}\left(e^{2 \pi i\left(t+\frac{2 k}{N}\right)}, x\right) \overline{Z \hat{g}\left(e^{2 \pi i\left(t+\frac{2 k+2 j}{N}\right)}, y\right)}\right. \\
& \left.+Z \hat{g}\left(e^{2 \pi i\left(t+\frac{2 k+1}{N}\right)}, x\right) \overline{Z \hat{g}\left(e^{2 \pi i\left(t+\frac{2 k+2 j+1}{N}\right)}, y\right)}\right] \\
= & \frac{4}{N^{2}} \sum_{x, y=0}^{\frac{N}{2}-1}\left[\sum_{k=0}^{\frac{N}{2}-1} e^{-2 \pi i \frac{2 k}{N}(x-y)}\right] e^{2 \pi i \frac{2 j}{N} y}\left[Z \hat{g}\left(e^{2 \pi i t}, x\right) \overline{Z \hat{g}\left(e^{2 \pi i t}, y\right)}\right. \\
& \left.+Z \hat{g}\left(e^{2 \pi i\left(t+\frac{1}{N}\right)}, x\right) \overline{Z \hat{g}\left(e^{2 \pi i\left(t+\frac{1}{N}\right)}, y\right)}\right] \\
= & \frac{2}{N} \sum_{x=0}^{\frac{N}{2}-1}\left[\left|Z \hat{g}\left(e^{2 \pi i t}, x\right)\right|^{2}+\left|Z \hat{g}\left(e^{2 \pi i\left(t+\frac{1}{N}\right)}, x\right)\right|^{2}\right] e^{2 \pi i \frac{2 j}{N} x} \\
= & \frac{2}{N}\left[\left|Z \hat{g}\left(e^{2 \pi i t}, \cdot\right)\right|^{2}+\left|Z \hat{g}\left(e^{2 \pi i\left(t+\frac{1}{N}\right)}, \cdot\right)\right|^{2}\right]^{\vee}(j),
\end{aligned}
$$

where the inverse Fourier transform is taken in $\mathbb{Z}_{\frac{N}{2}}$. This shows that (iii) is equivalent to

$$
\frac{2}{N}\left[\left|Z \hat{g}\left(e^{2 \pi i t}, \cdot\right)\right|^{2}+\left|Z \hat{g}\left(e^{2 \pi i\left(t+\frac{1}{N}\right)}, \cdot\right)\right|^{2}\right]^{\vee}(j)=N \delta_{j, 0}
$$

If (ii) holds, then

$$
\frac{2}{N}\left[\left|Z \hat{g}\left(e^{2 \pi i t}, \cdot\right)\right|^{2}+\left|Z \hat{g}\left(e^{2 \pi i\left(t+\frac{1}{N}\right)}, \cdot\right)\right|^{2}\right]^{\vee}(j)=\frac{2}{N} N \sum_{x=0}^{\frac{N}{2}-1} e^{2 \pi i \frac{2 j}{N} x}=N \delta_{j, 0},
$$

which is (3.4). On the other hand, the inverse Fourier transform is injective. This proves that (3.4) holds if and only if (ii) is true and thus (ii) $\Leftrightarrow$ (iii).

(iii) $\Leftrightarrow$ (iv): First we remark that $\mathcal{W}\left(g, \Lambda, \ell_{2}(\mathbb{Z})\right)$ is an orthonormal basis if and only if the set

$$
\Psi:=\left\{T_{n N} f_{m}: m=1, \ldots, N, n \in \mathbb{Z}\right\},
$$


where

$$
\begin{aligned}
f_{1}(x) & =g(x), \\
f_{N}(x) & =g_{0, \frac{N}{2}}(x), \\
f_{2 l+k}(x) & =\frac{(-1)^{k l}}{\sqrt{2}}\left(g_{k \frac{N}{2}, \frac{l}{N}}+(-1)^{k+l} g_{k \frac{N}{2},-\frac{l}{N}}\right), l=1, \ldots, \frac{N}{2}-1, k=0,1
\end{aligned}
$$

is an orthonormal basis, since these elements differ from the elements in $\mathcal{W}\left(g, \Lambda, \ell_{2}(\mathbb{Z})\right)$ only by factors of absolute value 1 . Next notice that to prove (iv) it is sufficient and necessary that

$$
\left\|T_{n N} f_{m}\right\|_{2}=1, \quad m=1, \ldots, L, n \in \mathbb{Z}
$$

and

$$
\sum_{m=1}^{L} \sum_{n \in \mathbb{Z}}\left\langle h_{1}, T_{n N} f_{m}\right\rangle\left\langle T_{n N} f_{m}, h_{2}\right\rangle=\left\langle h_{1}, h_{2}\right\rangle, \quad \text { for all } h_{1}, h_{2} \in \ell_{2}(\mathbb{Z}) .
$$

We start by dealing with (3.5). Using the Plancherel theorem, we compute

$$
\begin{gathered}
1=\left\|T_{n N} f_{1}\right\|_{2}^{2}=\|\hat{g}\|_{2}^{2}=\int_{0}^{1} \hat{g}\left(e^{2 \pi i t}\right) \hat{g}\left(e^{2 \pi i t}\right) d t, \\
1=\left\|T_{n N} f_{N}\right\|_{2}^{2}=\left\|\hat{g}_{\frac{N}{2}, 0}\right\|^{2}=\int_{0}^{1} \hat{g}\left(e^{2 \pi i t}\right) \hat{g}\left(e^{2 \pi i t}\right) d t,
\end{gathered}
$$

and, for $m=2, \ldots, N-1$,

$$
\begin{aligned}
1= & \left\|T_{n N} f_{m}\right\|_{2}^{2} \\
= & \| \frac{1}{\sqrt{2}}\left(\hat{g}_{\frac{l}{N},-k \frac{N}{2}}+(-1)^{k+l} \hat{g}_{\left.-\frac{l}{N},-k \frac{N}{2}\right)} \|_{2}^{2}\right. \\
= & \frac{1}{2} \int_{0}^{1}\left|e^{-2 \pi i \frac{k N}{2} t}\right|^{2}\left|\hat{g}\left(e^{2 \pi i\left(t-\frac{l}{N}\right)}\right)+(-1)^{k+l} \hat{g}\left(e^{2 \pi i\left(t+\frac{l}{N}\right)}\right)\right|^{2} d t \\
= & \frac{1}{2} \int_{0}^{1}\left[\left|\hat{g}\left(e^{2 \pi i\left(t-\frac{l}{N}\right)}\right)\right|^{2}+\left|\hat{g}\left(e^{2 \pi i\left(t+\frac{l}{N}\right)}\right)\right|^{2}+(-1)^{k+l} \hat{g}\left(e^{2 \pi i\left(t-\frac{l}{N}\right)}\right) \overline{\hat{g}\left(e^{2 \pi i\left(t+\frac{l}{N}\right)}\right)}\right. \\
& \left.+(-1)^{k+l} \overline{\hat{g}\left(e^{2 \pi i\left(t-\frac{l}{N}\right)}\right)} \hat{g}\left(e^{2 \pi i\left(t+\frac{l}{N}\right)}\right)\right] d t .
\end{aligned}
$$

Since $\hat{g}$ is real-valued, we can continue the last computation and obtain that

$$
1=\left\|T_{n N} f_{m}\right\|_{2}^{2}=\|\hat{g}\|_{2}^{2}+(-1)^{k+l} \int_{0}^{1} \hat{g}\left(e^{2 \pi i t}\right) \hat{g}\left(e^{2 \pi i\left(t+\frac{2 l}{N}\right)}\right) d t .
$$

Combining the above computations we have proven that (3.5) holds if and only if

$$
\int_{0}^{1} \hat{g}\left(e^{2 \pi i t}\right) \hat{g}\left(e^{2 \pi i\left(t+\frac{2 j}{N}\right)}\right) d t=\delta_{j, 0} \quad \text { for all } j \in\{0, \ldots, N-1\} .
$$

Now we turn to the study of condition (3.6). Using the Plancherel formula and the Poisson summation formula [18, Theorem 4.42] applied to $H=\left\{e^{2 \pi i \frac{k}{N}}: k=\right.$ $0, \ldots, N-1\}$, we obtain

$$
\sum_{m=1}^{N} \sum_{n \in \mathbb{Z}}\left\langle h_{1}, T_{n N} f_{m}\right\rangle\left\langle T_{n N} f_{m}, h_{2}\right\rangle
$$




$$
\begin{aligned}
& =\sum_{m=1}^{N} \sum_{n \in \mathbb{Z}}\left\langle\widehat{h_{1}}, \widehat{T_{n N} f_{m}}\right\rangle\left\langle\widehat{T_{n N} f_{m}}, \widehat{h_{2}}\right\rangle \\
& =\sum_{m=1}^{N} \sum_{n \in \mathbb{Z}} \int_{0}^{1}\left(\widehat{h_{1}} \overline{\widehat{f_{m}}}\right)\left(e^{2 \pi i t}\right) \int_{0}^{1}\left(\widehat{\widehat{f_{m}}} \overline{\widehat{h_{2}}}\right)\left(e^{2 \pi i s}\right) e^{-2 \pi i s N n} d s e^{2 \pi i t N n} d t \\
& =\sum_{m=1}^{N} \int_{0}^{1}\left(\widehat{\left(h_{1}\right.} \overline{\widehat{f_{m}}}\right)\left(e^{2 \pi i t}\right)\left[\sum_{n \in \mathbb{Z}}\left(\widehat{\left(f_{m}\right.} \overline{\widehat{h_{2}}}\right)^{\wedge}(N n) e^{2 \pi i t N n}\right] d t \\
& =\sum_{m=1}^{N} \int_{0}^{1}\left(\widehat{\left(h_{1}\right.} \overline{\widehat{f_{m}}}\right)\left(e^{2 \pi i t}\right) \frac{1}{N} \sum_{r=0}^{N-1}\left(\widehat{f_{m}} \overline{\widehat{h_{2}}}\right)\left(e^{2 \pi i\left(t+\frac{r}{N}\right)}\right) d t \text {, }
\end{aligned}
$$

which equals $\left\langle h_{1}, h_{2}\right\rangle$ if and only if

$$
\sum_{m=1}^{N} \widehat{\widehat{f_{m}}\left(e^{2 \pi i t}\right)} \widehat{f_{m}}\left(e^{2 \pi i\left(t+\frac{r}{N}\right)}\right)=N \delta_{r, 0} \quad \text { for all } r \in\{0, \ldots, N-1\} .
$$

Setting $\mathbb{L}:=\left\{-\frac{N}{2}+1, \ldots,-1,1, \ldots, \frac{N}{2}-1\right\}$, we compute

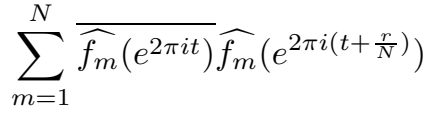

$$
\begin{aligned}
& =\hat{g}\left(e^{2 \pi i t}\right) \hat{g}\left(e^{2 \pi i\left(t+\frac{r}{N}\right)}\right)+\hat{g}_{\frac{N}{2}, 0}\left(e^{2 \pi i t}\right) \hat{g}_{\frac{N}{2}, 0}\left(e^{2 \pi i\left(t+\frac{r}{N}\right)}\right)+\frac{1}{2} \sum_{l=1}^{\frac{N}{2}-1} \sum_{k=0}^{1}\left[\overline{\hat{g}_{\frac{l}{N},-k \frac{N}{2}}\left(e^{2 \pi i t}\right)}\right.
\end{aligned}
$$

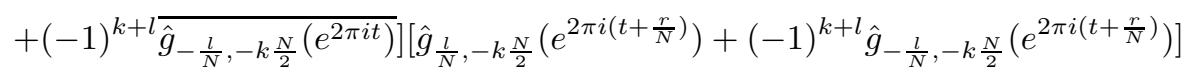

$$
\begin{aligned}
& =\hat{g}\left(e^{2 \pi i t}\right) \hat{g}\left(e^{2 \pi i\left(t+\frac{r}{N}\right)}\right)+\hat{g}\left(e^{2 \pi i\left(t-\frac{N}{2}\right)}\right) \hat{g}\left(e^{2 \pi i\left(t+\frac{r}{N}-\frac{N}{2}\right)}\right)+\frac{1}{2} \sum_{l=1}^{\frac{N}{2}-1} \sum_{k=0}^{1} e^{-2 \pi i k \frac{N}{2} \frac{r}{N}}\left[\hat{g}\left(e^{2 \pi i\left(t-\frac{l}{N}\right)}\right)\right. \\
& \left.+(-1)^{k+l} \hat{g}\left(e^{2 \pi i\left(t+\frac{l}{N}\right)}\right)\right]\left[\hat{g}\left(e^{2 \pi i\left(t-\frac{l}{N}+\frac{r}{N}\right)}\right)+(-1)^{k+l} \hat{g}\left(e^{2 \pi i\left(t+\frac{l}{N}+\frac{r}{N}\right)}\right)\right] \\
& =\hat{g}\left(e^{2 \pi i t}\right) \hat{g}\left(e^{2 \pi i\left(t+\frac{r}{N}\right)}\right)+\hat{g}\left(e^{2 \pi i\left(t-\frac{N}{2}\right)}\right) \hat{g}\left(e^{2 \pi i\left(t+\frac{r}{N}-\frac{N}{2}\right)}\right) \\
& +\frac{1}{2} \sum_{l=1}^{\frac{N}{2}-1} \sum_{k=0}^{1}(-1)^{k r}\left[\hat{g}\left(e^{2 \pi i\left(t-\frac{l}{N}\right)}\right) \hat{g}\left(e^{2 \pi i\left(t-\frac{l}{N}+\frac{r}{N}\right)}\right)+\hat{g}\left(e^{2 \pi i\left(t+\frac{l}{N}\right)}\right) \hat{g}\left(e^{2 \pi i\left(t+\frac{l}{N}+\frac{r}{N}\right)}\right)\right. \\
& \left.\left.+(-1)^{k+l}\left(e^{2 \pi i\left(t-\frac{l}{N}\right)}\right) \hat{g}\left(e^{2 \pi i\left(t+\frac{l}{N}+\frac{r}{N}\right)}\right)+\hat{g}\left(e^{2 \pi i\left(t+\frac{l}{N}\right)}\right) \hat{g}\left(e^{2 \pi i\left(t-\frac{l}{N}+\frac{r}{N}\right)}\right)\right)\right] \\
& =\hat{g}\left(e^{2 \pi i t}\right) \hat{g}\left(e^{2 \pi i\left(t+\frac{r}{N}\right)}\right)+\sum_{l \in \mathbb{L}} \hat{g}\left(e^{2 \pi i\left(t+\frac{l}{N}\right)}\right) \hat{g}\left(e^{2 \pi i\left(t+\frac{l}{N}+\frac{r}{N}\right)}\right)\left[\frac{1+(-1)^{r}}{2}\right] \\
& +\sum_{l \in \mathbb{L}}(-1)^{l} \hat{g}\left(e^{2 \pi i\left(t+\frac{l}{N}\right)}\right) \hat{g}\left(e^{2 \pi i\left(t-\frac{l}{N}+\frac{r}{N}\right)}\right)\left[\frac{1+(-1)^{r+1}}{2}\right]+\hat{g}\left(e^{2 \pi i\left(t-\frac{N}{2}\right)}\right) \hat{g}\left(e^{2 \pi i\left(t+\frac{r}{N}-\frac{N}{2}\right)}\right) .
\end{aligned}
$$

If $r$ is even, i.e., $r=2 j$, we obtain

$$
\sum_{m=1}^{N} \overline{\widehat{f_{m}}\left(e^{2 \pi i t}\right)} \widehat{f_{m}}\left(e^{2 \pi i\left(t+\frac{r}{N}\right)}\right)=\sum_{l=0}^{N-1} \hat{g}\left(e^{2 \pi i\left(t+\frac{l}{N}\right)}\right) \hat{g}\left(e^{2 \pi i\left(t+\frac{l}{N}+\frac{2 j}{N}\right)}\right),
$$


and if $r$ is odd, i.e., $r=2 j+1$, we obtain

$$
\begin{aligned}
& \sum_{m=1}^{N} \overline{\widehat{f_{m}}\left(e^{2 \pi i t}\right)} \widehat{f_{m}}\left(e^{2 \pi i\left(t+\frac{r}{N}\right)}\right) \\
& =\hat{g}\left(e^{2 \pi i t}\right) \hat{g}\left(e^{2 \pi i\left(t+\frac{r}{N}\right)}\right)+\hat{g}\left(e^{2 \pi i\left(t-\frac{N}{2}\right)}\right) \hat{g}\left(e^{2 \pi i\left(t+\frac{r}{N}-\frac{N}{2}\right)}\right) \\
& +\sum_{l \in \mathbb{L}}(-1)^{l} \hat{g}\left(e^{2 \pi i\left(t+\frac{l}{N}\right)}\right) \hat{g}\left(e^{2 \pi i\left(t-\frac{l}{N}+\frac{r}{N}\right)}\right) \\
& =0 \text {. }
\end{aligned}
$$

This shows that (3.6) holds if and only if

$$
\sum_{l=0}^{N-1} \hat{g}\left(e^{2 \pi i\left(t+\frac{l}{N}\right)}\right) \hat{g}\left(e^{2 \pi i\left(t+\frac{l+2 j}{N}\right)}\right)=N \delta_{j, 0} .
$$

Moreover, this equation implies equation (3.7), since

$$
\int_{0}^{1} \hat{g}\left(e^{2 \pi i t}\right) \hat{g}\left(e^{2 \pi i\left(t+\frac{2 j}{N}\right)}\right) d t=\int_{0}^{\frac{1}{N}} \sum_{l=0}^{N-1} \hat{g}\left(e^{2 \pi i\left(t+\frac{l}{N}\right)}\right) \hat{g}\left(e^{2 \pi i\left(t+\frac{l+2 j}{N}\right)}\right) d t=\int_{0}^{\frac{1}{N}} N \delta_{j, 0} d t=\delta_{j, 0} .
$$

This shows (iii) $\Leftrightarrow$ (iv), and hence the theorem is proven. $\square$

Now we will study the case of a general time-frequency lattice.

Proposition 3.6. Let $\Lambda$ be a lattice in $\mathbb{Z} \times \mathbb{T}$ with $\operatorname{vol}(\Lambda)=\frac{1}{2}$ with canonical generator matrix A given by

$$
A=\left[\begin{array}{cc}
\frac{N}{2} & b \\
0 & \frac{1}{N}
\end{array}\right] .
$$

Let $g \in \ell_{2}(\mathbb{Z})$, let $m_{0}, n_{0} \in \mathbb{Z}$ be chosen such that $\frac{N}{2} m_{0}+b n_{0}=\operatorname{gcd}\left(\frac{N}{2}, b\right)=: c$, and let $U$ be defined on $\ell_{2}(\mathbb{Z})$ by

$$
U f(k)=f(k) e^{\pi i \frac{n_{0}}{c N} k^{2}}
$$

Then

$$
g_{m \frac{N}{2}+n b, n \frac{1}{N}}(l)=C(m, n) U\left(U^{-1} g\right)_{m \frac{N}{2}+n b,-m \frac{n_{0}}{2 c}-n \frac{b n_{0}}{c N}+n \frac{1}{N}}(l), \quad l \in \mathbb{Z},
$$

where $C(m, n)=e^{\pi i \frac{n_{0}}{c N}\left(m \frac{N}{2}+n b\right)^{2}}$.

Proof. Let $\sigma \in \operatorname{Hom}(\mathbb{Z} \times \mathbb{T})$ be defined by

$$
\sigma=\left[\begin{array}{cc}
I_{\mathbb{Z}} & 0 \\
-\frac{n_{0}}{c N} & I_{\mathbb{T}}
\end{array}\right]
$$

It is easy to check that $\sigma$ is symplectic on $\mathbb{Z} \times \mathbb{T}$. In order to apply Theorem 1.1 we need to compute a second degree character of $\mathbb{Z} \times \mathbb{T}$ associated to $\zeta=\sigma^{*} \kappa_{0} \sigma-\kappa_{0}$, where $\kappa_{0}$ is defined by

$$
\kappa_{0}=\left[\begin{array}{cc}
1 & 1 \\
I_{\mathbb{Z}} & 0
\end{array}\right] \in \operatorname{Hom}(\mathbb{Z} \times \mathbb{T}, \mathbb{T} \times \mathbb{Z})
$$

First we note that $\sigma^{*}(z, n)=\left(z e^{2 \pi i\left(-\frac{n_{0}}{c N}\right) n}, n\right)$, since

$$
\left\langle(m, t), \sigma^{*}(z, n)\right\rangle=\langle\sigma(m, t),(z, n)\rangle=z^{m}\left(e^{2 \pi i\left(-\frac{n_{0}}{c N}\right) m} t\right)^{n}=\left\langle(m, t),\left(z e^{2 \pi i\left(-\frac{n_{0}}{c N}\right) n}, n\right)\right\rangle .
$$


Thus

$$
\zeta(n, z)=\left(\sigma^{*} \kappa_{0} \sigma-\kappa_{0}\right)(n, z)=\sigma^{*} \kappa_{0}\left(n, e^{2 \pi i\left(-\frac{n_{0}}{c N}\right) n} z\right)-(1, n)=\left(e^{2 \pi i\left(-\frac{n_{0}}{c N}\right) n}, 0\right) .
$$

The map

$$
\psi: \mathbb{Z} \times \mathbb{T} \rightarrow \mathbb{T}, \quad \psi(m, t)=e^{-\pi i \frac{n_{0}}{c N} m^{2}}
$$

is a second degree character associated to $\zeta$ as the following calculation shows:

$$
\begin{aligned}
\psi(m, t) \psi(n, z)\langle(m, t), \zeta(n, z)\rangle & =e^{-\pi i \frac{n_{0}}{c N} m^{2}} e^{-\pi i \frac{n_{0}}{c N} n^{2}} e^{2 \pi i\left(-\frac{n_{0}}{c N}\right) m n} \\
& =e^{-\pi i \frac{n_{0}}{c N}(m+n)^{2}} \\
& =\psi((m, t)+(n, z)) .
\end{aligned}
$$

Next notice that

$$
\sigma\left(m \frac{N}{2}+n b, n \frac{1}{N}\right)=\left(m \frac{N}{2}+n b,-m \frac{n_{0}}{2 c}-n \frac{b n_{0}}{c N}+n \frac{1}{N}\right) .
$$

Now we can apply Theorem 1.1 which proves the claim.

Next we define a Wilson basis associated with a lattice with arbitrary canonical generator matrix. For this, the following mapping will turn out to be very useful.

Lemma 3.7. Let $\frac{N}{2}, b \in \mathbb{Z}$ with $0 \leq b<\frac{N}{2}$, and let $m_{0}, n_{0} \in \mathbb{Z}$ be chosen such that $\frac{N}{2} m_{0}+b n_{0}=\operatorname{gcd}\left(\frac{N}{2}, b\right)=: c$. Further let $d:=\operatorname{lcm}\left(\frac{N}{2}, b\right)$. Then the mapping $\varphi: \mathbb{Z}^{2} \rightarrow \mathbb{Z}^{2}$ defined by

$$
\varphi(m, n)=\left\{\begin{array}{cc}
(m, n) & : \quad b=0 \\
\left(m m_{0}-\frac{2 d}{N} n, m n_{0}+\frac{d}{b} n\right) & : \quad b \neq 0
\end{array}\right.
$$

is bijective and, for all $m \in \mathbb{Z}$, we have

$$
\left\{(m, n \bmod 2 c):(m, n) \in \varphi^{-1}(\mathbb{Z} \times\{0, \ldots, N-1\})\right\}=\{m\} \times\{0, \ldots, 2 c-1\}
$$

with

$$
\left|\left\{n:(m, n) \in \varphi^{-1}(\mathbb{Z} \times\{0, \ldots, N-1\})\right\}\right|=2 c .
$$

Proof. We only need to study the case $b \neq 0$. For this, let $(m, n),\left(m^{\prime}, n^{\prime}\right) \in \mathbb{Z}^{2}$ be such that $\varphi(m, n)=\varphi\left(m^{\prime}, n^{\prime}\right)$. Then

$$
\frac{N}{2}\left(m m_{0}-\frac{2 d}{N} n\right)+b\left(m n_{0}+\frac{d}{b} n\right)=\frac{N}{2}\left(m^{\prime} m_{0}-\frac{2 d}{N} n^{\prime}\right)+b\left(m^{\prime} n_{0}+\frac{d}{b} n^{\prime}\right),
$$

which holds if and only if

$$
m\left(\frac{N}{2} m_{0}+b n_{0}\right)=m^{\prime}\left(\frac{N}{2} m_{0}+b n_{0}\right),
$$

and hence $m=m^{\prime}$. This implies

$$
\left(-\frac{2 d}{N} n, \frac{d}{b} n\right)=\left(-\frac{2 d}{N} n^{\prime}, \frac{d}{b} n^{\prime}\right),
$$

which yields $n=n^{\prime}$. This proves that $\varphi$ is injective.

To show that $\varphi$ is surjective, let $(k, l) \in \mathbb{Z}^{2}$ and consider $M:=\frac{N}{2} k+b l$. It is well-known that there exists some $m \in \mathbb{Z}$ with $M=m c$. Furthermore, we have

$$
\left\{(p, q) \in \mathbb{Z}^{2}: \frac{N}{2} p+b q=m c\right\}=\left\{\left(m m_{0}-\frac{2 d}{N} n, m n_{0}+\frac{d}{b} n\right): n \in \mathbb{Z}\right\},
$$


since $\frac{N}{2} p+b q=\frac{N}{2} p^{\prime}+b q^{\prime}$ if and only if $\frac{N}{2}\left(p-p^{\prime}\right)=b\left(q^{\prime}-q\right)$. This yields the existence of some $n \in \mathbb{Z}$ with

$$
\varphi(m, n)=\left(m m_{0}-\frac{2 d}{N} n, m n_{0}+\frac{d}{b} n\right)=(k, l) .
$$

Secondly, we will prove the second part of the lemma. First observe that $m, n \in \mathbb{Z}$ satisfy

$$
\varphi(m, n) \in \mathbb{Z} \times\{0, \ldots, N-1\}
$$

if and only if they satisfy

$$
-\frac{b}{d} n_{0} m \leq n \leq-\frac{b}{d} n_{0} m+\frac{b}{d}(N-1)=2 c-\frac{b}{d} n_{0} m-\frac{b}{d} .
$$

Hence, for each fixed $m \in \mathbb{Z}$, the set of $n \in \mathbb{Z}$ such that (3.10) is satisfied equals

$$
S_{m}:=\left\{\left\lceil-\frac{b}{d} n_{0} m\right\rceil, \ldots,\left\lfloor 2 c-\frac{b}{d} n_{0} m-\frac{b}{d}\right\rfloor\right\} .
$$

To finish the proof we claim that

$$
\left|S_{m}\right|=2 c \quad \text { for all } m \in \mathbb{Z} .
$$

For this, fix $m \in \mathbb{Z}$ and let $k \in \mathbb{Z}$ and $l \in\left\{0, \ldots, \frac{d}{b}-1\right\}$ be such that $-n_{0} m=k \frac{d}{b}+l$. Then we obtain $\left\lceil-\frac{b}{d} n_{0} m\right\rceil=k$ if $l=0$ and otherwise $\left\lceil-\frac{b}{d} n_{0} m\right\rceil=k+1$. Moreover, we have

$$
\left\lfloor 2 c-\frac{b}{d} n_{0} m-\frac{b}{d}\right\rfloor=\left\lfloor 2 c+k+\frac{l-1}{\frac{d}{b}}\right\rfloor,
$$

which equals $2 c+k-1$ if $l=0$ and otherwise $2 c+k$. Thus the second part of the lemma is proven. $\square$

Note that the following definition reduces to Definition 3.4 in the case of a diagonal canonical generator matrix.

Definition 3.8. Let $\Lambda$ be a lattice in $\mathbb{Z} \times \mathbb{T}$ with canonical generator matrix $A$ given by

$$
A=\left[\begin{array}{cc}
\frac{N}{2} & b \\
0 & \frac{1}{N}
\end{array}\right] .
$$

Let $g \in \ell_{2}(\mathbb{Z})$, and let $\varphi$ be defined as in Lemma 3.7. Then the Wilson system $\mathcal{W}\left(g, \Lambda, \ell_{2}(\mathbb{Z})\right)=\left\{\psi_{m, n}\right\}_{m \in \mathbb{Z}, n=0, \ldots, \frac{N}{2}}$ is given by

$$
\psi_{m, n}^{\Lambda}=g_{\varphi_{1}(2 m, n) \frac{N}{2}+\varphi_{2}(2 m, n) b, \varphi_{2}(2 m, n) \frac{1}{N}}, \quad \text { if } m \in \mathbb{Z}, n=0, \frac{N}{2},
$$

and for $m \in \mathbb{Z}, n=1, \ldots, \frac{N}{2}-1$,

$$
\begin{aligned}
\psi_{m, n}^{\Lambda}= & \frac{1}{\sqrt{2}}\left(g_{\varphi_{1}(m, n) \frac{N}{2}+\varphi_{2}(m, n) b, \varphi_{2}(m, n) \frac{1}{N}}\right. \\
& +g_{\left.\varphi_{1}(m,-n) \frac{N}{2}+\varphi_{2}(m,-n) b, \varphi_{2}(m,-n) \frac{1}{N}\right),} \text { if } m+n \text { even }, \\
\psi_{m, n}^{\Lambda}= & \frac{i}{\sqrt{2}}\left(g_{\varphi_{1}(m, n) \frac{N}{2}+\varphi_{2}(m, n) b, \varphi_{2}(m, n) \frac{1}{N}}\right. \\
& -g_{\left.\varphi_{1}(m,-n) \frac{N}{2}+\varphi_{2}(m,-n) b, \varphi_{2}(m,-n) \frac{1}{N}\right),} \text { if } m+n \text { odd } .
\end{aligned}
$$


The following theorem gives an equivalent condition for a Wilson system with respect to an arbitrary time-frequency lattice to form an orthonormal basis in terms of a frame condition for the associated Gabor system.

ThEOREM 3.9. Let $\Lambda$ be a lattice in $\mathbb{Z} \times \mathbb{T}$ with canonical generator matrix $A$ given by

$$
A=\left[\begin{array}{cc}
\frac{N}{2} & b \\
0 & \frac{1}{N}
\end{array}\right]
$$

Let $g \in \ell_{2}(\mathbb{Z})$ be such that $\widehat{U^{-1} g}$ is real-valued, let $M:=2 c$, and let $U$ and $\varphi$ be defined as in Proposition 3.6 and Lemma 3.7. respectively. Then the following conditions are equivalent.

(i) $\left\{g_{m \frac{N}{2}+n b, n \frac{1}{N}}\right\}_{m \in \mathbb{Z}, n=0, \ldots, N-1}$ is a tight frame for $\ell_{2}(\mathbb{Z})$ with frame bound 2.

(ii) $\left\{\left(U^{-1} g\right)_{m \frac{M}{2}, n \frac{1}{M}}\right\}_{m \in \mathbb{Z}, n=0, \ldots, M-1}$ is a tight frame for $\ell_{2}(\mathbb{Z})$ with frame bound 2.

(iii) $\mathcal{W}\left(U^{-1} g, \frac{M}{2} \mathbb{Z} \times \frac{1}{M}\{0, \ldots, M-1\}, \ell_{2}(\mathbb{Z})\right)$ is an orthonormal basis for $\ell_{2}(\mathbb{Z})$.

(iv) $\mathcal{W}\left(g, \Lambda, \ell_{2}(\mathbb{Z})\right)$ is an orthonormal basis for $\ell_{2}(\mathbb{Z})$.

Proof. Let $\sigma$ be defined as in the proof of Proposition 3.6 and let $\varphi=\left(\varphi_{1}, \varphi_{2}\right)$. Then we compute

$$
\begin{aligned}
\sigma & \left(\varphi_{1}(m, n) \frac{N}{2}+\varphi_{2}(m, n) b, \varphi_{2}(m, n) \frac{1}{N}\right) \\
= & \left(\varphi_{1}(m, n) \frac{N}{2}+\varphi_{2}(m, n) b,-\varphi_{1}(m, n) \frac{n_{0}}{2 c}-\varphi_{2}(m, n) \frac{b n_{0}}{c N}+\varphi_{2}(m, n) \frac{1}{N}\right) \\
= & m\left(\frac{N}{2} m_{0}+b n_{0}\right)+n\left(-\frac{2 d}{N} \frac{N}{2}+\frac{d}{b} b\right), m\left(-\frac{n_{0}}{2 c} m_{0}-\frac{b n_{0}}{c N} n_{0}+\frac{1}{N} n_{0}\right) \\
& \left.+n\left(\frac{2 d}{N} \frac{n_{0}}{2 c}-\frac{d}{b} \frac{b n_{0}}{c N}+\frac{d}{b} \frac{1}{N}\right)\right) \\
= & \left(m c, m n_{0}\left(\frac{1}{N}-\frac{1}{c N}\left(\frac{N}{2} m_{0}+b n_{0}\right)\right)+n \frac{d}{b N}\right) \\
= & \left(m \frac{M}{2}, n \frac{1}{M}\right),
\end{aligned}
$$

where in the last step we used $c d=\frac{N}{2} b$. Using Lemma 3.7 the equivalence of (i) and (ii) now follows immediately from Proposition 3.6 and Equation (3.9), since $U$ is unitary and $|C(m, n)|=1$. Proposition 3.5proves (ii) $\Leftrightarrow$ (iii). Therefore it remains to prove the equivalence of (iii) and (iv). For this, we will use the following implication of Proposition 3.6

$$
\begin{aligned}
U\left(U^{-1} g\right)_{m \frac{M}{2}, n \frac{1}{M}} & =U\left(U^{-1} g\right)_{\sigma\left(\varphi_{1}(m, n) \frac{N}{2}+\varphi_{2}(m, n) b, \varphi_{2}(m, n) \frac{1}{N}\right)} \\
& =C(\varphi(m, n))^{-1} g_{\varphi_{1}(m, n) \frac{N}{2}+\varphi_{2}(m, n) b, \varphi_{2}(m, n) \frac{1}{N}} .
\end{aligned}
$$

Further notice that $C(\varphi(m, n))^{-1}$ does not depend on the sign of $n$, since

$$
C(\varphi(m, n))^{-1}=e^{-\pi i \frac{n_{0}}{c N}\left(\varphi_{1}(m, n) \frac{N}{2}+\varphi_{2}(m, n) b\right)^{2}}=e^{-\pi i \frac{n_{0}}{c N} m^{2}\left(m_{0} \frac{N}{2}+n_{0} b\right)^{2}}=e^{-\pi i \frac{n_{0}}{N} m^{2} c^{2}} .
$$

Using now the definition of a Wilson basis, the fact that $U$ is a unitary operator, and the fact that $|C(\varphi(m, n))|=1$ yields the result. $\mathrm{Q}$

4. Wilson bases for general lattices - the finite case. The space $\mathbb{C}^{L}$ has several advantages over $\ell^{2}(\mathbb{Z})$ when constructing numerical methods for practical timefrequency analysis, which often allow a further acceleration of numerical algorithms, e.g., see [1, 31].

Before defining Gabor systems and Wilson systems for $\mathbb{C}^{L}$ for general timefrequency lattices, we first prove that each such lattice does not only possess a uniquely 
determined generator matrix in Hermite normal form (which was already proved in [22]), but moreover in our situation this matrix attains a special form.

Lemma 4.1. Let $\Lambda$ be a lattice in $\mathbb{Z}_{L} \times \mathbb{Z}_{L}$ with generator matrix $A$ given by

$$
A=\left[\begin{array}{ll}
a & b \\
c & d
\end{array}\right], \quad \text { with } a, b, c, d \in \mathbb{N}, \text { and } \operatorname{det}(A)=\frac{L}{2},
$$

and denote $p=\operatorname{gcd}(c, d)$ if $c \neq 0$ and $p=d$ if $c=0$. Then $\Lambda$ possesses a uniquely determined generator matrix of the form

$$
A^{\prime}=\left[\begin{array}{cc}
\frac{L}{2 p} & b^{\prime} \\
0 & p
\end{array}\right]
$$

where $p=\operatorname{gcd}(c, d)$ and $0 \leq b^{\prime}<\frac{L}{2 p}$.

Proof. Let $p=\operatorname{gcd}(c, d)$ with $c=q p, d=r p$ and note that $p \mid \frac{L}{2} \operatorname{since} \operatorname{det}(A)=$ $a d-b c=(a r-b q) p=\frac{L}{2}$. Since $\frac{d}{p}=r$ and $\frac{-c}{p}=-q$, we have

$$
A\left[\begin{array}{c}
r \\
-q
\end{array}\right]=\left[\begin{array}{c}
\frac{L}{2 p} \\
0
\end{array}\right] \text {. }
$$

Furthermore, we claim that there exists a $z \in \mathbb{Z}$ with $0 \leq z<\frac{L}{2 p}$ such that the point $\left[\begin{array}{l}z \\ p\end{array}\right]$ belongs to $\Lambda$. This can be seen as follows: The condition $\left[\begin{array}{l}z \\ p\end{array}\right] \in \Lambda$ is equivalent to the existence of $m, n \in \mathbb{Z}$ such that

$$
\left[\begin{array}{ll}
a & b \\
c & d
\end{array}\right]\left[\begin{array}{l}
m \\
n
\end{array}\right]=\left[\begin{array}{l}
z \\
p
\end{array}\right]
$$

Consider the equation $c m+d n=p$ and substitute $c=q p, d=r p$, then $q p m+r p n=p$, hence $q m+r n=1$. Since $r$ and $q$ are relative prime, there exist $m, n \in \mathbb{Z}$ such that $q m+r n=1$ (see e.g. [24] Theorem 4.4]). Thus (4.3) holds for $z \in \mathbb{Z}$, but we still have to show that it holds under the condition $0 \leq z<\frac{L}{2 p}$. We can write $z=b^{\prime}+k \frac{L}{2 p}$ with $0 \leq b^{\prime}<\frac{L}{2 p}$ and $k \in \mathbb{Z}$. Hence

$$
\left[\begin{array}{l}
z \\
p
\end{array}\right]=\left[\begin{array}{c}
b^{\prime} \\
p
\end{array}\right]+k\left[\begin{array}{c}
\frac{L}{2 p} \\
0
\end{array}\right]
$$

Since $\left[\begin{array}{c}\frac{L}{2 p} \\ 0\end{array}\right]=A\left[\begin{array}{c}r \\ -q\end{array}\right]$ by (4.2), it follows that $\left[\begin{array}{c}b^{\prime} \\ p\end{array}\right] \in \Lambda$. Consequently the matrix

$$
A^{\prime}=\left[\begin{array}{cc}
\frac{L}{2 p} & b^{\prime} \\
0 & p
\end{array}\right]
$$

(which satisfies $\operatorname{det}\left(A^{\prime}\right)=\frac{L}{2}$ ) generates $\Lambda$.

The fact that this matrix is uniquely determined is an immediate consequence from the condition $0 \leq b^{\prime}<\frac{L}{2 p}$.

Definition 4.2. Let $\Lambda$ be a lattice in $\mathbb{Z}_{L} \times \mathbb{Z}_{L}$. Then the uniquely determined matrix $A^{\prime}$ of Lemma 4.1 is called the canonical generator matrix for $\Lambda$. 
Using the notion of a canonical generator matrix, we first give the definition of a Gabor system.

DeFINITION 4.3. Let $\Lambda$ be a lattice in $\mathbb{Z}_{L} \times \mathbb{Z}_{L}$ with canonical generator matrix A given by

$$
A=\left[\begin{array}{cc}
\frac{L}{2 p} & b \\
0 & p
\end{array}\right]
$$

Set $M=2 p, N=\frac{L}{p}$ and let $g$ be some L-periodic function on $\mathbb{Z}$. Then the associated Gabor system is given by $\left\{g_{m a+n b, n d}\right\}_{m=0, \ldots, M-1, n=0, \ldots, N-1}$, where

$$
g_{m a+n b, n d}(l)=g(l-(m a+n b)) e^{2 \pi i l n d / L}, \quad l=0, \ldots, L-1 .
$$

Next we define a Wilson basis associated with a lattice with diagonal canonical generator matrix in the following way:

DEFINITION 4.4. Let $\Lambda$ be a lattice in $\mathbb{Z}_{L} \times \mathbb{Z}_{L}$ with canonical generator matrix A given by

$$
A=\left[\begin{array}{cc}
\frac{L}{2 p} & 0 \\
0 & p
\end{array}\right]
$$

and let $g$ be some $L$-periodic function on $\mathbb{Z}$. Then the Wilson system $\mathcal{W}\left(g, \Lambda, \mathbb{C}^{L}\right)=$ $\left\{\psi_{m, n}\right\}_{(m, n) \in I}$, where $I=\{0, \ldots, p-1\} \times\left\{0, \frac{L}{2 p}\right\} \cup\{0, \ldots, 2 p-1\} \times\left\{1, \ldots, \frac{L}{2 p}-1\right\}$, is given by

$$
\psi_{m, n}^{\Lambda}=g_{m \frac{L}{p}, n p}, \quad \text { if } m=0, \ldots, p-1, n=0, \frac{L}{2 p},
$$

and for $m=0, \ldots, 2 p-1, n=1, \ldots, \frac{L}{2 p}-1$,

$$
\begin{array}{ll}
\psi_{m, n}^{\Lambda}=\frac{1}{\sqrt{2}}\left(g_{m \frac{L}{2 p}, n p}+g_{m \frac{L}{2 p},-n p}\right), & \text { if } m+n \text { even }, \\
\psi_{m, n}^{\Lambda}=\frac{i}{\sqrt{2}}\left(g_{m \frac{L}{2 p}, n p}-g_{m \frac{L}{2 p},-n p}\right), & \text { if } m+n \text { odd } .
\end{array}
$$

Also in the finite case we will employ the Zak transform. This time we will use the Zak transform on the group $\mathbb{Z}_{L}$ with respect to the uniform lattice $K=\{2 p k$ : $\left.k=0, \ldots, \frac{L}{2 p}-1\right\}$ in $\mathbb{Z}_{L}$, which is defined on the set of square-integrable functions on the set $\{0, \ldots, 2 p-1\} \times\left\{0, \ldots, \frac{L}{2 p}-1\right\}$ by

$$
Z f(x, y)=\sum_{k=0}^{\frac{L}{2 p}-1} f(x+2 p k) e^{2 \pi i \frac{2 p k}{L} y},
$$

where we associate $\mathbb{Z}_{L}$ with $\{0, \ldots, L-1\}$.

The following proposition is the analog to Proposition 3.5 for the space $\mathbb{C}^{L}$.

Proposition 4.5. Let $g$ be some $L$-periodic function on $\mathbb{Z}$ such that $\hat{g}$ is realvalued and consider the lattice $\Lambda$ with canonical generator matrix given by

$$
\left[\begin{array}{cc}
\frac{L}{2 p} & 0 \\
0 & p
\end{array}\right]
$$

Then the following conditions are equivalent. 
(i) $\left\{g_{m \frac{L}{2 p}, n p}\right\}_{m=0, \ldots, 2 p-1, n=0, \ldots, \frac{L}{p}-1}$ is a tight frame for $\mathbb{C}^{L}$ with frame bound 2 .

(ii) We have $|Z \hat{g}(x, y)|^{2}+|Z \hat{g}(x+p, y)|^{2}=\frac{1}{p}$ a.e..

(iii) For all $j=0, \ldots, \frac{L}{2 p}-1$ and $y \in \mathbb{Z}_{L}$, we have $\sum_{l=0}^{\frac{L}{p}-1} \hat{g}(y+l p) \hat{g}(y+l p+2 j p)=$ $\frac{1}{p} \delta_{j, 0}$.

(iv) $\mathcal{W}\left(g, \Lambda, \mathbb{C}^{L}\right)$ is an orthonormal basis for $\mathbb{C}^{L}$

Proof. The proof, while lengthy, is very similar to the proof of Proposition 3.5 In fact, with obvious adaptations, such as using the normalized Haar measure on $\mathbb{Z}_{L}$, i.e., $m(E)=\frac{1}{L} \sum_{x \in \mathbb{Z}_{L}} 1_{E}(x)$ for all $E \subseteq \mathbb{Z}_{L}$, and replacing Zak transforms and Fourier transforms by their corresponding finite counterparts, the proof carries over almost line by line. We therefore leave this part to the reader.

Now we will turn our attention to general time-frequency lattices. Here the situation is slightly more involved compared to $\ell^{2}(\mathbb{Z})$.

Let $\Lambda$ be a lattice in $\mathbb{Z}_{L} \times \mathbb{Z}_{L}$ with canonical generator matrix $A$ given by

$$
A=\left[\begin{array}{cc}
\frac{L}{2 p} & b \\
0 & p
\end{array}\right] .
$$

Then we choose $\alpha, \beta, m_{0}, n_{0} \in \mathbb{Z}$ such that

$$
\alpha \frac{L}{2 p} m_{0}+\alpha b n_{0}+\beta p n_{0}
$$

attains its minimal positive value. Assume that there exists a choice of $\alpha, \beta, m_{0}, n_{0}$ such that $\left(\frac{L}{2 p} m_{0}+b n_{0}\right)\left(p n_{0}\right)<0$ and $\left(\alpha \frac{L}{2 p}\right)(\alpha b+\beta p)>0$. In the other cases we have to change the signs of the later defined $\gamma$ and $\delta$ accordingly. In the following we will restrict to the case where $|\alpha|=1$. For the remainder of this section let $\alpha, \beta, m_{0}, n_{0}$ be defined in this way. Now we regard $\alpha$ and $\beta$ as elements of $\mathbb{Z}_{L}$. For the sake of brevity we set

$$
c:=\operatorname{gcd}\left(\alpha \frac{L}{2 p}, \alpha b+\beta p\right), \quad d:=\operatorname{lcm}\left(\alpha \frac{L}{2 p}, \alpha b+\beta p\right),
$$

and

$$
s:=\operatorname{gcd}\left(\frac{L}{2 p} m_{0}+b n_{0}, p n_{0}\right), \quad t:=\operatorname{lcm}\left(\frac{L}{2 p} m_{0}+b n_{0}, p n_{0}\right) .
$$

The minimality condition for (4.4) shows that

$$
\alpha \frac{L}{2 p} m_{0}+\alpha b n_{0}+\beta p n_{0}=c=s .
$$

We further define $\gamma, \delta \in \mathbb{Z}_{L}$ by

$$
\gamma:=\frac{t}{\frac{L}{2 p} m_{0}+b n_{0}} \quad \text { and } \quad \delta:=-\frac{t}{p n_{0}}
$$

and $\sigma \in \operatorname{Hom}\left(\mathbb{Z}_{L} \times \mathbb{Z}_{L}\right)$ by

$$
\sigma=\left[\begin{array}{ll}
\alpha & \beta \\
\gamma & \delta
\end{array}\right]
$$

Proposition 4.6. Let $\Lambda$ be a lattice in $\mathbb{Z}_{L} \times \mathbb{Z}_{L}$ with canonical generator matrix A given by

$$
A=\left[\begin{array}{cc}
\frac{L}{2 p} & b \\
0 & p
\end{array}\right]
$$


Let $\sigma$ be defined as in the preceding paragraph, and let $U$ on the space of $L$-periodic functions on $\mathbb{Z}$ be defined by

$$
U f(k)=\sum_{l \in \mathbb{Z}_{L}} f(\alpha k+\beta l) e^{-\pi i\left(\alpha \gamma k^{2}+\beta \delta l^{2}\right)(L+1) / L} e^{-2 \pi i \beta \gamma k l / L} .
$$

Then

$$
g_{m \frac{L}{2 p}+n b, n p}(l)=C(m, n) U\left(U^{-1} g\right)_{\sigma\left(m \frac{L}{2 p}+n b, n p\right)}(l),
$$

where $C(m, n)=e^{-\pi i\left(\alpha \gamma m^{2}+\beta \delta n^{2}\right)(L+1) / L} e^{-2 \pi i \beta \gamma m n / L}$.

Before moving on to the proof of this statement we point out that the operator $U$ in Proposition 4.6 is no longer a simple chirp operator as for the case $\ell^{2}(\mathbb{Z})$, cf. Proposition 3.6 This difference and the different form of $\sigma$ necessitates a somewhat different proof for the case $\mathbb{C}^{L}$.

Proof. In order to apply Theorem 1.1 we need to check whether $\sigma=\left(\sigma_{1}, \sigma_{2}\right)$ is symplectic. For this, we have to show that, for all $(x, y),\left(x^{\prime}, y^{\prime}\right) \in \mathbb{Z}_{L} \times \mathbb{Z}_{L}$,

$$
e^{-2 \pi i \sigma_{2}(x, y) \sigma_{1}\left(x^{\prime}, y^{\prime}\right) / L} e^{2 \pi i \sigma_{2}\left(x^{\prime}, y^{\prime}\right) \sigma_{1}(x, y) / L}=e^{-2 \pi i x^{\prime} y / L} e^{2 \pi i x y^{\prime} / L} .
$$

We have

$$
\begin{aligned}
& e^{-2 \pi i \sigma_{2}(x, y) \sigma_{1}\left(x^{\prime}, y^{\prime}\right) / L} e^{2 \pi i \sigma_{2}\left(x^{\prime}, y^{\prime}\right) \sigma_{1}(x, y) / L} \\
& =e^{-2 \pi i(\gamma x+\delta y)\left(\alpha x^{\prime}+\beta y^{\prime}\right) / L} e^{2 \pi i\left(\gamma x^{\prime}+\delta y^{\prime}\right)(\alpha x+\beta y) / L} \\
& =e^{2 \pi i(\alpha \delta-\beta \gamma)\left(x y^{\prime}-x^{\prime} y\right) / L}
\end{aligned}
$$

and

$$
\begin{aligned}
\alpha \delta-\beta \gamma & =-\frac{\alpha t}{p n_{0}}-\frac{\beta t}{\frac{L}{2 p} m_{0}+b n_{0}} \\
& =\frac{-t}{\left(\frac{L}{2 p} m_{0}+b n_{0}\right)\left(p n_{0}\right)}\left(\alpha\left(\frac{L}{2 p} m_{0}+b n_{0}\right)+\beta p n_{0}\right) .
\end{aligned}
$$

By (4.5),

$$
\alpha \delta-\beta \gamma=\frac{-s t}{\left(\frac{L}{2 p} m_{0}+b n_{0}\right)\left(p n_{0}\right)}=1
$$

since $\left(\frac{L}{2 p} m_{0}+b n_{0}\right)\left(p n_{0}\right)<0$. This proves that (4.6) is satisfied, which shows that $\sigma$ is indeed symplectic. Moreover, a short computation analogous to the one in the proof of Proposition 3.6 shows that

$$
\zeta(k, l)=\left(\sigma^{*} \kappa_{0} \sigma-\kappa_{0}\right)(k, l)=(\alpha \gamma k+\beta \gamma l, \beta \gamma k+\beta \delta l) .
$$

Now it is easy to check (compare also [26, Example 1.1.34 (iii)]) that

$$
\psi: \mathbb{Z}_{L}^{2} \rightarrow \mathbb{T}, \quad \psi(k, l)=e^{\pi i\left(\alpha \gamma k^{2}+\beta \delta l^{2}\right)(L+1) / L} e^{2 \pi i \beta \gamma k l / L}
$$

is a second degree character associated to $\zeta$. Applying Theorem 1.1 now finishes the proof. 
As in the discrete case we need to define a special bijective map in order to give the definition of a Wilson basis associated with a lattice with arbitrary canonical generator matrix.

LeMma 4.7. Let $\frac{L}{2 p}, b \in \mathbb{Z}_{L}$ with $0 \leq b<\frac{L}{2 p}$ and let $\alpha, \beta, m_{0}, n_{0}, c, d$ be defined as before. Then the mapping $\varphi: \mathbb{Z}^{2} \rightarrow \mathbb{Z}^{2}$ defined by

$$
\varphi(m, n)=\left\{\begin{array}{cc}
(m, n) & : \quad b=0 \\
\left(m m_{0}-\frac{2 p d}{\alpha L} n, m n_{0}+\frac{d}{\alpha b+\beta p} n\right) & : \quad b \neq 0
\end{array}\right.
$$

is bijective and we have

$$
\begin{gathered}
\left\{\left(m \bmod \frac{L}{c}, n \bmod 2 c\right):(m, n) \in \varphi^{-1}\left(\{0, \ldots, 2 p-1\} \times\left\{0, \ldots, \frac{L}{p}-1\right\}\right)\right\} \\
=\left\{0, \ldots, \frac{L}{c}-1\right\} \times\{0, \ldots, 2 c-1\} .
\end{gathered}
$$

Proof. The proof of this lemma is very similar to the proof of Lemma 3.7 we therefore omit it.

Note that the following definition reduces to Definition 4.4 in the case of a diagonal canonical generator matrix.

Definition 4.8. Let $\Lambda$ be a lattice in $\mathbb{Z}_{L} \times \mathbb{Z}_{L}$ with canonical generator matrix A given by

$$
A=\left[\begin{array}{cc}
\frac{L}{2 p} & b \\
0 & p
\end{array}\right] .
$$

Let $g$ be some $L$-periodic function on $\mathbb{Z}$, and let $\varphi$ be defined as in Lemma 4.7. Then the Wilson system $\mathcal{W}\left(g, \Lambda, \mathbb{C}^{L}\right)=\left\{\psi_{m, n}\right\}_{(m, n) \in I}$, where $I=\{0, \ldots, p-1\} \times\left\{0, \frac{L}{2 p}\right\} \cup$ $\{0, \ldots, 2 p-1\} \times\left\{1, \ldots, \frac{L}{2 p}-1\right\}$, is given by

$$
\psi_{m, n}^{\Lambda}=g_{\varphi_{1}(2 m, n) \frac{L}{2 p}+\varphi_{2}(2 m, n) b, \varphi_{2}(2 m, n) p}, \quad \text { if } m=0, \ldots, p-1, n=0, \frac{L}{2 p},
$$

and for $m=0, \ldots, 2 p-1, n=1, \ldots, \frac{L}{2 p}-1$,

$$
\begin{aligned}
\psi_{m, n}^{\Lambda}= & \frac{1}{\sqrt{2}}\left(g_{\varphi_{1}(m, n) \frac{L}{2 p}+\varphi_{2}(m, n) b, \varphi_{2}(m, n) p}\right. \\
& \left.+g_{\varphi_{1}(m,-n) \frac{L}{2 p}+\varphi_{2}(m,-n) b, \varphi_{2}(m,-n) p}\right), \quad \text { if } m+n \text { even }, \\
\psi_{m, n}^{\Lambda}= & \frac{i}{\sqrt{2}}\left(g_{\varphi_{1}(m, n) \frac{L}{2 p}+\varphi_{2}(m, n) b, \varphi_{2}(m, n) p}\right. \\
& \left.-g_{\varphi_{1}(m,-n) \frac{L}{2 p}+\varphi_{2}(m,-n) b, \varphi_{2}(m,-n) p}\right), \quad \text { if } m+n \text { odd } .
\end{aligned}
$$

The following theorem is the analog to Theorem 3.9 for the space $\mathbb{C}^{L}$.

THEOREM 4.9. Let $\Lambda$ be a lattice in $\mathbb{Z}_{L} \times \mathbb{Z}_{L}$ with canonical generator matrix $A$ given by

$$
A=\left[\begin{array}{cc}
\frac{L}{2 p} & b \\
0 & p
\end{array}\right]
$$

Let $g$ be some $L$-periodic function on $\mathbb{Z}$ such that $\widehat{U^{-1} g}$ is real-valued, let $M:=2 p$, $N:=\frac{L}{p}, q:=\frac{L}{2 c}, \tilde{M}:=2 q, \tilde{N}:=\frac{L}{q}$, and let $U$ and $\varphi$ be defined as in Proposition 4.6 and Lemma 4.7, respectively. Then the following conditions are equivalent. 
(i) $\left\{g_{m \frac{L}{2 p}+n b, n p}\right\}_{m=0, \ldots, M-1, n=0, \ldots, N-1}$ is a tight frame for $\mathbb{C}^{L}$ with frame bound 2.

(ii) $\left\{\left(U^{-1} g\right)_{m \frac{L}{2 q}, n q}\right\}_{m=0, \ldots, \tilde{M}-1, n=0, \ldots, \tilde{N}-1}$ is a tight frame for $\mathbb{C}^{L}$ with frame bound 2 .

(iii) $\mathcal{W}\left(U^{-1} g, \frac{L}{2 q} \mathbb{Z}_{L} \times q \mathbb{Z}_{L}, \mathbb{C}^{L}\right)$ is an orthonormal basis for $\mathbb{C}^{L}$.

(iv) $\mathcal{W}\left(g, \Lambda, \mathbb{C}^{L}\right)$ is an orthonormal basis for $\mathbb{C}^{L}$.

Proof. Let $\sigma$ be defined as before and let $\varphi=\left(\varphi_{1}, \varphi_{2}\right)$. Then we compute

$$
\begin{aligned}
\sigma & \left.\varphi_{1}(m, n) \frac{L}{2 p}+\varphi_{2}(m, n) b, \varphi_{2}(m, n) p\right) \\
= & \left(\alpha\left(\varphi_{1}(m, n) \frac{L}{2 p}+\varphi_{2}(m, n) b\right)+\beta \varphi_{2}(m, n) p, \gamma\left(\varphi_{1}(m, n) \frac{L}{2 p}+\varphi_{2}(m, n) b\right)+\delta \varphi_{2}(m, n) p\right) \\
= & \left(m\left(\alpha \frac{L}{2 p} m_{0}+\alpha b n_{0}+\beta p n_{0}\right)+n\left(-\alpha \frac{L}{2 p} \frac{2 p d}{\alpha L}+\alpha b \frac{d}{\alpha b+\beta p}+\beta p \frac{d}{\alpha b+\beta p}\right),\right. \\
& \left.m\left(\gamma \frac{L}{2 p} m_{0}+\gamma b n_{0}+\delta p n_{0}\right)+n\left(-\gamma \frac{L}{2 p} \frac{2 p d}{\alpha L}+\gamma b \frac{d}{\alpha b+\beta p}+\delta p \frac{d}{\alpha b+\beta p}\right)\right) \\
= & \left(m c, m\left(\frac{t}{\frac{L}{2 p} m_{0}+b n_{0}}\left(\frac{L}{2 p} m_{0}+b n_{0}\right)-\frac{t}{p n_{0}} p n_{0}\right)\right. \\
& \left.+n\left(-\frac{t}{\frac{L}{2 p} m_{0}+b n_{0}} \frac{d}{\alpha}+\frac{t}{\frac{L}{2 p} m_{0}+b n_{0}} \frac{b d}{\alpha b+\beta p}-\frac{t}{p n_{0}} \frac{p d}{\alpha b+\beta p}\right)\right) \\
= & \left(m c, n t d\left(\frac{-\alpha b p n_{0}-\beta p^{2} n_{0}+\alpha b p n_{0}-\alpha p \frac{L}{2 p} m_{0}-\alpha p b n_{0}}{\alpha\left(\frac{L}{2 p} m_{0}+b n_{0}\right)(\alpha b+\beta p) p n_{0}}\right)\right) \\
= & \left(m c,-n t d p\left(\frac{\alpha \frac{L}{2 p} m_{0}+\alpha b n_{0}+\beta p n_{0}}{\alpha\left(\frac{L}{2 p} m_{0}+b n_{0}\right)(\alpha b+\beta p) p n_{0}}\right)\right) \\
= & \left.\left(m c,-n t p \frac{c d}{\alpha\left(\frac{L}{2 p} m_{0}+b n_{0}\right)(\alpha b+\beta p) p n_{0}}\right)\right) \\
= & \left(m c,-n t \frac{\frac{L}{2}}{\left(\frac{L}{2 p} m_{0}+b n_{0}\right) p n_{0}}\right) \\
= & \left.\left(m c, n \frac{L}{2 c}\right)\right),
\end{aligned}
$$

where in the last step we used (4.5) and $s t=-\left(\frac{L}{2 p} m_{0}+b n_{0}\right) p n_{0}$. Since $|\alpha|=1$, we have $c=s=\operatorname{gcd}\left(\alpha \frac{L}{2 p}, \alpha b+\beta p\right)$ is a factor of $\frac{L}{2 p}$ and hence of $\frac{L}{2}$. Using Lemma 4.7 the equivalence of (i) and (ii) follows immediately from Proposition 4.6 since $U$ is unitary and $|C(m, n)|=1$. Proposition 4.5 proves (ii) $\Leftrightarrow$ (iii). Therefore it remains to prove the equivalence of (iii) and (iv). For this, we will use the following implication of Proposition 4.6

$$
\begin{aligned}
U\left(U^{-1} g\right)_{m \frac{L}{2 q}, n q} & =U\left(U^{-1} g\right)_{\sigma\left(\varphi_{1}(m, n) \frac{L}{2 p}+\varphi_{2}(m, n) b, \varphi_{2}(m, n) p\right)} \\
& =C(\varphi(m, n))^{-1} g_{\varphi_{1}(m, n) \frac{L}{2 p}+\varphi_{2}(m, n) b, \varphi_{2}(m, n) p} .
\end{aligned}
$$

An easy but tedious calculation shows that $C(\varphi(m, n))^{-1}$ does not depend on the sign of $n$. Using now the definition of a Wilson basis, the fact that $U$ is a unitary operator, and the fact that $|C(\varphi(m, n))|=1$ yields the result. $\mathbf{C}$

Tight Gabor frames in $\mathbb{C}^{L}$ can be constructed in the same way as for $\ell^{2}(\mathbb{Z})$ and $L^{2}(\mathbb{R})$ by using the "inverse square root trick". Furthermore, it has been shown in 27$]$ that for properly localized windows the dual window constructed in $\mathbb{C}^{L}$ by "sampling and periodization" of the frame $\left\{g_{m a, n b}\right\}$ converges to the dual window $S^{-1} g$ with increasing sampling rate and increasing periodization interval, see 27] for details. This result can be easily extended to tight windows. We refer also to [25, 32, 10, for related results and leave the details to the reader. To obtain tight Gabor frames in $\mathbb{C}^{L}$ that satisfy the required conditions of the theorem above and have good timefrequency localization one can thus essentially proceed analogous to the example at the end of Section 2 
5. Conclusion. We have demonstrated that orthonormal Wilson bases for $L^{2}(\mathbb{R})$ (with excellent time-frequency localization) can be constructed for general time-frequency lattices. Of course any numerical implementation has to be done in a discrete setting. Somewhat longer proofs establish a similar result for the spaces $\ell^{2}(\mathbb{Z})$ and $\mathbb{C}^{L}$ for non-rectangular time-frequency lattices. The approach based on metaplectic transforms used in this paper suggests that the main results can be extended to the setting of symplectic time-frequency lattices on general locally compact abelian groups.

Furthermore, our results imply that from a practical viewpoint it is indeed possible to extend OQAM-OFDM or cosine-modulated filter banks to general time-frequency lattices. Moreover, we expect that the benefits of using general time-frequency lattices will be even more pronounced for images and higher-dimensional signals. Our expectation is based on the fact that in the theory of sphere packings (and sphere coverings) the advantages of the optimal sphere packing over the packing associated with the rectangular lattices increases significantly with the dimension of the space [8].

An interesting research problem is thus to investigate how to extend the results in this paper to $L^{2}\left(\mathbb{R}^{d}\right)$ for non-symplectic lattices as well as to find optimal timefrequency lattices in $\mathbb{R}^{2 d}$ for $d>1$. One possibility to define an "optimal" timefrequency lattice is to fix the function $g$ to be a Gaussian, say, and then find that time-frequency lattice of fixed density which minimizes the condition number of the associated Gabor frame operator as indicated in 33.

Acknowledgments. We thank the referees for valuable comments and suggestions which lead to an improvement of the results and presentation in this paper.

\section{REFERENCES}

[1] L. Auslander and R. Tolimieri, On finite Gabor expansions of signals, in Signal Processing, Part I: Signal Processing Theory, L. Auslander, T. Kailath, and S.K. Mitter, eds., Springer Verlag, New York, 1990, pp. 13-23, IMA vol. 22, lectures from IMA Program, summer 1988.

[2] J.J. Benedetto, W. Czaja, And A.Y. Maltsev, The Balian-Low theorem for the symplectic form on $\mathbb{R}^{2 d}$, J. Math. Phys., 44 (2003), pp. 1735-1750.

[3] J.J. Benedetto, C. Heil, And D.F. Walnut, Differentiation and the Balian-Low theorem, J. Four. Anal. Appl., 1 (1995), pp. 355-403.

[4] K. Bittner, Wilson bases on the interval, in Advances in Gabor Analysis, H.G. Feichtinger and T. Strohmer, eds., Appl. Numer. Harmon. Anal., Birkhäuser, Boston, MA, 2003, pp. 197221.

[5] H. BöLCSKEI, Orthogonal frequency division multiplexing based on offset QAM, in Advances in Gabor Analysis, H.G. Feichtinger and T. Strohmer, eds., Birkhäuser, Boston, MA, 2002, pp. 321-352.

[6] H. Bölcskei, H.G. Feichtinger, K. Gröchenig, and F. Hlawatsch. Discrete-time Wilson expansions. Proc. IEEE Int. Sympos. Time-Frequency Time-Scale Analysis, Paris (France), pp. 525-528, June 1996.

[7] H. BÖlcskei and F. Hlawatsch, Oversampled modulated filter banks, in Gabor Analysis and Algorithms: Theory and Applications, H.G. Feichtinger and T. Strohmer, eds., Birkhäuser, Boston, MA, 1998, pp. 295-322.

[8] J.H. Conway and N.J.A. Sloane, editors. Sphere Packings, Lattices and Groups. Grundlehren der mathematischen Wissenschaften. Springer Verlag, New York, Berlin, Heidelberg, 1993.

[9] O. Christensen, An introduction to frames and Riesz bases, Birkhäuser, Boston, 2003.

[10] O. Christensen and T. Strohmer. Methods for approximation of the inverse (Gabor) frame operator. In Advances in Gabor analysis, pages 171-195. Birkhäuser Boston, Boston, MA, 2003.

[11] I. Daubechies, S. Jaffard, And J.L. Journé, A simple Wilson orthonormal basis with exponential decay, SIAM J. Math. Anal., 22 (1991), pp. 554-572. 
[12] H.G. Feichtinger, O. Christensen, And T. Strohmer, A group-theoretical approach to Gabor analysis, Optical Engineering, 34 (1995), pp. 1697-1704.

[13] H.G. FeICHTINGER AND W. KOZEK, Quantization of TF-lattice invariant operators on elementary LCA groups, in Gabor Analysis and Algorithms: Theory and Applications, H.G. Feichtinger and T. Strohmer, eds., Birkhäuser, Boston, MA, 1998, pp. 233-266.

[14] H.G. Feichtinger and T. Strohmer, eds., Gabor Analysis and Algorithms: Theory and Applications, Birkhäuser, Boston, 1998.

[15] H.G. Feichtinger and T. Strohmer, eds., Advances in Gabor analysis, Birkhäuser Boston Inc., Boston, MA, 2003.

[16] B. Le Floch, M. Alard, and C. Berrou, Coded orthogonal frequency division multiplex, Proc. of IEEE, 83 (1995), pp. 982-996.

[17] G.B. Folland, Harmonic Analysis in Phase Space, Annals of Math, Studies. Princeton Univ. Press, Princeton (NJ), 1989.

[18] G.B. Folland, A course in abstract harmonic analysis, CRC Press, Baco Raton, 1995.

[19] K. Gröchenig, Aspects of Gabor analysis on locally compact abelian groups, in Gabor Analysis and Algorithms: Theory and Applications, H.G. Feichtinger and T. Strohmer, eds., Birkhäuser, Boston, MA, 1998, pp. 211-231.

[20] K. Gröchenig, Foundations of Time-Frequency Analysis, Birkhäuser, Boston, 2001.

[21] K. Gröchenig, D. Han, C. Heil, and G. Kutyniok, The Balian-Low theorem for symplectic lattices in higher dimensions, Appl. Comp. Harm. Anal., 13 (2002), pp. 169-176.

[22] C. Hermite, Extraits de lettres de M. Ch. Hermite à M. Jacobi sur differents objets de la théorie des nombres, Deuxième lettre, Reine Angewandte Mathematik, 40 (1850), pp. 279290.

[23] E. HewitT And K.A. Ross, Abstract harmonic analysis I, II, Springer-Verlag, Berlin/Heidelberg/New York, 1963/1970.

[24] L.K. HuA, Introduction to number theory, Springer-Verlag, Berlin, 1982.

[25] A.J.E.M. Janssen. From continuous to discrete Weyl-Heisenberg frames through sampling. J. Fourier Anal. Appl., 3(5):583-596, 1997.

[26] N. Kaiblinger, Metaplectic representation, eigenfunctions of phase space shifts, and GelfandShilov spaces for lca groups, Ph.D. thesis, University of Vienna, 1999.

[27] N. Kaiblinger, Approximation of the Fourier transform and the dual Gabor window, J. Fourier Anal. Appl., to appear.

[28] E. Kaniuth and G. Kutyniok, Zeros of the Zak transform on locally compact abelian groups, Proc. Amer. Math. Soc. 126 (1998), 3561-3569.

[29] W. Kozek And A. Molisch, Nonorthogonal pulseshapes for multicarrier communications in doubly dispersive channels, IEEE J. Sel. Areas Comm., 16 (1998), pp. 1579-1589.

[30] G. KUTYNIOK, Time-frequency analysis on locally compact groups, Ph.D. thesis, University of Paderborn, 2000.

[31] T. Strohmer, Numerical algorithms for discrete Gabor expansions, in Gabor Analysis and Algorithms: Theory and Applications, H.G. Feichtinger and T. Strohmer, eds., Birkhäuser, Boston, MA, 1998, pp. 267-294.

[32] T. Strohmer, Approximation of dual Gabor frames, window decay, and wireless communications, Appl. Comp. Harm. Anal., 11 (2001), pp. 243-262.

[33] T. Strohmer and S. Beaver, Optimal OFDM system design for time-frequency dispersive channels, IEEE Trans. Comm., 51:7 (2003), pp. 1111-1122.

[34] R. Tolimieri And M. An, Time-frequency representations, Applied and Numerical Harmonic Analysis, Birkhäuser Boston Inc., Boston, MA, 1998.

[35] K.G. WiLson, Generalized Wannier functions, (1987), preprint. 